\title{
Single- $W$ Production and Fermion-Loop Scheme: Numerical Results
}

\author{
Giampiero Passarino \\ Dipartimento di Fisica Teorica, Università di Torino, Italy \\ INFN, Sezione di Torino, Italy
}

\begin{abstract}
The single- $W$ production mechanism is synonymous to the $e^{+} e^{-}$annihilation into $e \nu_{e}$ and a $W$ boson with the outgoing electron lost in a small cone around the beam direction. It requires a Renormalization Scheme that preserves gauge invariance and fermion masses cannot be neglected in the calculation. A recently proposed generalization of the so-called Fermion-Loop scheme is applied to the evaluation of observables at LEP 2 energies. The total contribution to single- $W$ processes can be split, in a gauge invariant manner, into a $s$-channel component and a $t$-channel one. The latter is dominated by a regime of low momentum transfer of the outgoing electron and any high-energy Renormalization Scheme, as the $G_{F}$-one, fails to give the correct description of the scale. The Fermion-Loop scheme automatically converts, among other things, all couplings of the theory into couplings that are running at the appropriate scale. Therefore, in addition to represent the only scheme fully justified on a field-theoretical basis, the Fermion-Loop is the best starting point to include radiative corrections into single- $W$ production. Numerical results are presented, showing a decrease in the predictions that can be sizeable. There is no naive and overall rescaling of $\alpha_{\mathrm{QED}}$ in any pragmatic scheme, as the Fixed-Width one, that can reproduce the Fermion-Loop results, at the requested accuracy, for all configurations and for all kinematical cuts.
\end{abstract}

Pacs: 11.15.-q, 13.10.-q, 13.38.-b, 13.40.-f, 14.70.Fm 


\section{Introduction}

An interesting process at LEP 2 is the so-called single- $W$ production, $e^{+} e^{-} \rightarrow W e \nu$ which can be seen as a part of the CC20 process, $e^{+} e^{-} \rightarrow \bar{q} q\left(\mu \nu_{\mu}, \tau \nu_{\tau}\right) e \nu_{e}$, or as a part of the Mix56 process, $e^{+} e^{-} \rightarrow e^{+} e^{-} \nu_{e} \bar{\nu}_{e}$. For a theoretical review we refer to [1] and to [2]. For the experimental aspects we refer to the work of Ref. [3].

The CC20 process is usually considered in two regimes, $\left|\cos \theta\left(e^{-}\right)\right| \geq c$ or LACC20 and $\left|\cos \theta\left(e^{-}\right)\right| \leq$ $c$ or SACC20. Strictly speaking the single $W$ production is defined by those events that satisfy $\left|\cos \theta\left(e^{-}\right)\right| \geq 0.997$ and, therefore is a SACC20.

The LACC20 cross-section has been computed by many authors and references can be found in [4]. It represents a contribution to the $e^{+} e^{-} \rightarrow W^{+} W^{-}$total cross-section, in turn used to derive a value for $M_{W}$, the $W$ boson mass. This point deserves a comment: by $e^{+} e^{-} \rightarrow W^{+} W^{-}$it is meant the ideal cross-section obtained with the three double-resonant CC03 diagrams, see Fig. 1, and therefore the background, i.e. the full cross-section minus the CC03 one, is evaluated with the help of some MonteCarlo, estimating the error on the subtraction by comparing with some other MonteCarlo. Then $M_{W}$ is derived from a fit to $\sigma(\mathrm{CC} 03)$ with the help of a third calculation. From a theoretical point of view the evaluation of LACC20 is free of ambiguity, even in the approximation of massless fermions, as long as a gauge-preserving scheme is applied and $\theta\left(e^{-}\right)$is not too small.

For SACC20 instead, one cannot employ the massless approximation anymore and this fact makes the calculation much more difficult. In other words, in addition to double-resonant $W$-pair production with one $W$ decaying into $e \nu_{e}$, there are $t$-channel diagrams that give a sizeable contribution for small values of the polar scattering angle of the $t$-channel electron.

Single- $W$ processes are sensitive to the breaking of $\mathrm{U}(1)$ gauge invariance in the collinear limit, as described in Ref. [5] (see also [6]). The correct way of handling them is based on the so-called Fermion-Loop (FL)scheme [7], the gauge-invariant treatment of the finite-width effects of $W$ and $Z$ bosons in LEP2 processes. However, till very recently, the Fermion-Loop scheme was available only for the LACC20 regime.

For $e^{+} e^{-} \rightarrow e^{-} \bar{\nu}_{e} f_{1} \bar{f}_{2}$, the $\mathrm{U}(1)$ gauge invariance becomes essential in the region of phase space where the angle between the incoming and outgoing electrons is small, see the work of [5] and also an alternative formulations in [8]. In this limit the superficial $1 / Q^{4}$ divergence of the propagator structure is reduced to $1 / Q^{2}$ by $\mathrm{U}(1)$ gauge invariance. In the presence of light fermion masses this gives raise to the familiar $\ln \left(m_{e}^{2} / s\right)$ large logarithms.

Furthermore, keeping a finite electron mass through the calculation is not enough. One of the main results of [1] was to show that there are remaining subtleties in CC20, associated with the zero mass limit for the remaining fermions.

In Ref. [9] (hereafter I) a generalization of the Fermion-Loop scheme has been given (for previous work we refer to [10]). It consists of the re-summation of the fermionic one-loop corrections to the vector-boson propagators and of the inclusion of all remaining fermionic one-loop corrections, in particular those to the Yang-Mills vertices. In the original formulation, the Fermion-Loop scheme requires that vector bosons couple to conserved currents, i.e., that the masses of all external fermions can be neglected. There are several examples where fermion masses must be kept to obtain a reliable prediction. As already stated, this is the case for the single- $W$ production mechanism, where the outgoing electron is collinear, within a small cone, with the incoming electron. Therefore, $m_{e}$ cannot be neglected. 
Furthermore, among the 20 Feynman diagrams that contribute (for $e \bar{\nu}_{e} u \bar{d}$ final states, up to 56 for $\left.e^{+} e^{-} \nu_{e} \bar{\nu}_{e}\right)$ there are multi-peripheral ones that require a non-vanishing mass also for the other outgoing fermions. In I a generalization of the Fermion-Loop scheme is introduced to account for external, non-conserved, currents. Dyson re-summed transitions are introduced without neglecting the $p_{\mu} p_{\nu}$-terms and including the contributions from the Higgs-Kibble ghosts in the 't Hooft-Feynman gauge. In I we have introduced running vector boson masses and studied their relation with the corresponding complex poles. Always in I it is shown that any $\mathcal{S}$-matrix element takes a very simple form when written in terms of running masses. Finally, the relevant Ward identity, the U(1) Ward identity for single- $W$, is derived in the situation of interest, when all currents are non-conserved and when the top quark mass is not neglected inside loops.

For all details concerning the formal construction of the fully massive Fermion-Loop scheme we, therefore, refer to [9]. Here, instead, we concentrate on its implementation within the FORTRAN program WTO [11], on the corresponding numerical results and on the comparison, for single- $W$, between the Fermion-Loop (hereafter FL) scheme and the Fixed-Width (hereafter FW) one.

To be specific the name of Fixed-Width scheme is reserved for the following: the cross-section is computed using the tree-level amplitude. The massive gauge-boson propagators are given by $1 /\left(p^{2}+\right.$ $\left.m^{2}-i \Gamma\right)$. This gives an unphysical width for $p^{2}>0$, but retains $U(1)$ gauge invariance in the CC20 process.

The most recent numerical results produced for single- $W$ production are from the following codes [12]: CompHEP, GRC4F, NEXTCALIBUR, I PVALPHA, WPHACT and WTO.

Among these codes, WPHACT is the only one to employ the Fermion-Loop scheme in its imaginary version [14], where the full imaginary part of the Fermion-Loop corrections is used.

In view of a requested, inclusive cross-section, accuracy of $2 \%$ we must include radiative corrections to the best of our knowledge, at least the bulk of any large effect. As we know, the correct scale of the couplings and their differentiation between $s$ and $t$-channel is connected to the real part of the corrections, so that the imaginary FL is not enough, we need a complete FL for single- $W$. Having all the parts, the tree-level couplings are replaced by running couplings at the appropriate momenta and the massive gauge-boson propagators are modified accordingly. The vertex coefficients, entering through the Yang-Mills vertex, contain the lowest order couplings as well as the one-loop fermionic vertex corrections.

Apart from some recent development, each calculation aimed to provide some estimate for $e^{+} e^{-} \rightarrow$ $4 \mathrm{f}$ production is, at least nominally, a tree level calculation. Among other things it will require the choice of some Input Parameter Set (IPS) and of certain relations among the parameters. In the literature, although improperly, this is usually referred to as the choice of the Renormalization Scheme.

Typically, we have at our disposal four experimental data points (plus $\alpha_{s}$ ), i.e. the measured vector boson masses $M_{Z}, M_{W}$ and the coupling constants, $G_{F}$ and $\alpha$. However, we only have three bare parameters at our disposal, the charged vector boson mass, the $S U(2)$ coupling constant and the sinus of the weak mixing angle. While the inclusion of one loop corrections would allow us to fix at least the value of the top quark mass from a consistency relation, this cannot be done at the tree level. Thus, different choices of the basic relations among the input parameters can lead to different results with deviations which, in some case, can be sizeable.

\footnotetext{
${ }^{1}$ NEXTCALIBUR with masses, uses a massive matrix element program called HELAC, that is a recursive algorithm for electroweak amplitude calculations based on Dyson-Schwinger equations developed by A. Kanaki and C .G. Papadopou$\operatorname{los} 13]$.
} 
For instance, a possible choice is to fix the coupling constant $g$ as

$$
g^{2}=\frac{4 \pi \alpha}{s_{W}^{2}}, \quad s_{W}^{2}=\frac{\pi \alpha}{\sqrt{2} G_{F} M_{W}^{2}}
$$

where $G_{F}$ is the Fermi coupling constant. Another possibility would be to use

$$
g^{2}=4 \sqrt{2} G_{F} M_{W}^{2}
$$

but, in both cases, we miss the correct running of the coupling. Ad hoc solutions should be avoided, and the running of the parameters must always follow from a fully consistent scheme. Therefore, the only satisfactory solution can be found in the extension of the full Fermion-Loop to having nonzero external masses, or non-conserved currents. Unless, of course, one can compute the full set of corrections.

Note that the Fixed-Width scheme behaves properly in the collinear and high-energy regions of phase space, to the contrary of the Running-Width scheme, but it completely misses the running of the couplings, an effect that is expected to be above the requested precision tag of $2 \%$. By considering the impact of the FL-scheme on the relevant observables we will be able to judge on the goodness of naive rescaling procedures.

\section{The ingredients in the Fermion-Loop scheme.}

There are several building blocks that enter into the construction of the Fermion-Loop scheme, see for instance [15]. In the 't Hooft-Feynman gauge, the $\delta_{\mu \nu}$ part of the vector-vector transitions can be cast in the following form [15], where $s_{\theta}\left(c_{\theta}\right)$ is the sine(cosine) of the weak mixing angle:

$$
\begin{aligned}
S_{\gamma \gamma} & =\frac{g^{2} s_{\theta}^{2}}{16 \pi^{2}} \Pi_{\gamma \gamma}\left(p^{2}\right) p^{2}, \quad S_{Z Z}=\frac{g^{2}}{16 \pi^{2} c_{\theta}^{2}} \Sigma_{z Z}\left(p^{2}\right), \\
S_{Z \gamma} & =\frac{g^{2} s_{\theta}}{16 \pi^{2} c_{\theta}} \Sigma_{z \gamma}\left(p^{2}\right), \quad S_{W W}=\frac{g^{2}}{16 \pi^{2}} \Sigma_{W W}\left(p^{2}\right) .
\end{aligned}
$$

Next we have can transform to the $(3, Q)$ basis [15], where one writes

$$
\begin{aligned}
& \Sigma_{Z Z}\left(p^{2}\right)=\Sigma_{33}\left(p^{2}\right)-2 s_{\theta}^{2} \Sigma_{3 Q}\left(p^{2}\right)+s_{\theta}^{4} \Pi_{\gamma \gamma}\left(p^{2}\right) p^{2}, \\
& \Sigma_{Z \gamma}\left(p^{2}\right)=\Sigma_{3 Q}\left(p^{2}\right)-s_{\theta}^{2} \Pi_{\gamma \gamma}\left(p^{2}\right) p^{2} .
\end{aligned}
$$

We now consider three parameters, the e.m. coupling constant $e$, the $S U(2)$ coupling constant $g$ and the sine of the weak mixing angle $s_{\theta}$. At the tree level they are not independent, but rather they satisfy the relation $g^{2} s_{\theta}^{2}=e^{2}$. The running of the e.m. coupling constant is easily derived and gives

$$
\frac{1}{e^{2}(s)}=\frac{1}{g^{2} s_{\theta}^{2}}-\frac{1}{16 \pi^{2}} \Pi_{\gamma \gamma}(s)
$$

However, we have a natural scale to use since at $s=0$ we have the fine structure constant at our disposal. Therefore, the running of $e^{2}(s)$ is completely specified in terms of $\alpha$ by

$$
\frac{1}{e^{2}(s)}=\frac{1}{4 \pi \alpha}\left[1-\frac{\alpha}{4 \pi} \Pi(s)\right], \quad \text { with } \quad \Pi(s)=\Pi_{\gamma \gamma}(s)-\Pi_{\gamma \gamma}(0) .
$$



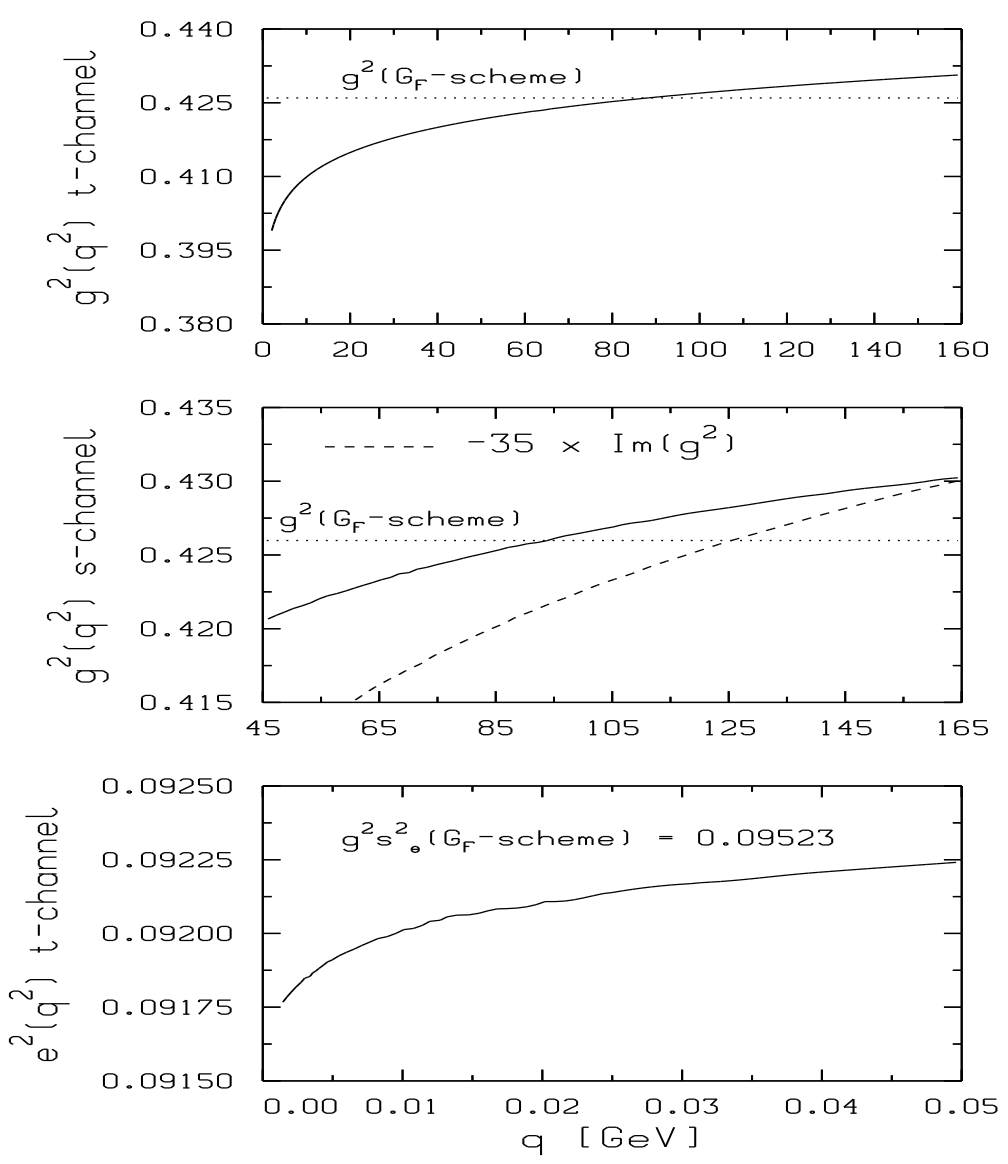

Figure 1: Running of coupling constants. The sign of $\operatorname{Im} g^{2}(s)$ is reversed and the corresponding curve is magnified by a factor 35. Here $q$ is the absolute value of the momentum.

For the running of $g^{2}$ we derive a similar equation:

$$
\frac{1}{g^{2}(s)}=\frac{1}{g^{2}}-\frac{1}{16 \pi^{2}} \Pi_{3 Q}(s)
$$

The running of the third parameter, $s_{\theta}^{2}(s)$, is now fixed by

$$
s_{\theta}^{2}(s)=\frac{e^{2}(s)}{g^{2}(s)} .
$$

In Fig. 1 1 we show the running of $e^{2}\left(q^{2}\right)$ for $q^{2} \rightarrow 0_{+}$, compared with the fixed value of $e^{2}$ that one would use in the $G_{F}$-scheme. Furthermore, in the same figure, we show the evolution of $g^{2}\left(q^{2}\right)$ for $q^{2}$ time-like or space-like, again compared with $g_{G_{F}}^{2}$. The sizeable difference that one gets between $e^{2}$ running in the $t$-channel and $e^{2}$ fixed in the $G_{F}$-scheme is expected to be one of the major improvements induced by the full Fermion-Loop scheme.

The re-summed propagators for the vector bosons are:

$$
G_{\gamma}\left(p^{2}\right)=\left\{p^{2}-S_{\gamma \gamma}\left(p^{2}\right)-\frac{\left[S_{z \gamma}\left(p^{2}\right)\right]^{2}}{p^{2}+M_{0}^{2}-S_{Z Z}\left(p^{2}\right)}\right\}^{-1}
$$




$$
\begin{aligned}
G_{Z \gamma}\left(p^{2}\right) & =\frac{S_{Z \gamma}\left(p^{2}\right)}{\left[p^{2}-S_{\gamma \gamma}\left(p^{2}\right)\right]\left[p^{2}+M_{0}^{2}-S_{Z Z}\left(p^{2}\right)\right]-\left[S_{Z \gamma}\left(p^{2}\right)\right]^{2}} \\
G_{Z}\left(p^{2}\right) & =\left\{p^{2}+M_{0}^{2}-S_{Z Z}-\frac{\left[S_{Z \gamma}\left(p^{2}\right)\right]^{2}}{p^{2}-S_{\gamma \gamma}\left(p^{2}\right)}\right\}^{-1}, \\
G_{W}\left(p^{2}\right) & =\left[p^{2}+M^{2}-S_{W W}\left(p^{2}\right)\right]^{-1} .
\end{aligned}
$$

The quantity $M_{0}=M / c_{\theta}$ is the bare $Z$ mass. An essential ingredient in the construction of the scheme is represented by the location of the complex poles. Substituting the corresponding results into the expressions for the propagators, Eq.([9), we see that all ultraviolet divergences not proportional to $p^{2}$ cancel. We obtain

$$
\begin{aligned}
G_{Z}(s) & =\left[-s+p_{Z}-Z(s)+Z\left(p_{Z}\right)\right]^{-1}, \\
G_{W}(s) & =\left[-s+p_{W}-S_{W W}(s)+S_{W W}\left(p_{W}\right)\right]^{-1}, \\
G_{Z \gamma}(s) & =-\frac{S_{Z \gamma}(s)}{s+S_{\gamma \gamma}(s)} G_{Z}(s), \\
G_{\gamma}(s) & =-\frac{1}{s+S_{\gamma \gamma}(s)}+\left[\frac{S_{Z \gamma}(s)}{s+S_{\gamma \gamma}(s)}\right]^{2} G_{Z}(s) .
\end{aligned}
$$

The vector boson propagators are now expressed as

$$
\begin{aligned}
G_{W}(s) & =-\frac{g^{2}(s)}{g^{2}} \frac{\omega_{W}(s)}{s}, \quad G_{Z}(s)=-\frac{c_{\theta}^{2}}{g^{2}} \frac{g^{2}(s)}{c^{2}(s)} \frac{\omega_{Z}(s)}{s}, \\
G_{Z \gamma}(s) & =\frac{s_{\theta}}{c_{\theta}}\left[1-\frac{s^{2}(s)}{s_{\theta}^{2}}\right] G_{Z}(s) \\
G_{\gamma}(s) & =\frac{e^{2}(s)}{e^{2}}+\frac{s_{\theta}^{2}}{c_{\theta}^{2}}\left[1-\frac{s^{2}(s)}{s_{\theta}^{2}}\right]^{2} G_{Z}(s),
\end{aligned}
$$

where the propagation functions are

$$
\begin{aligned}
& \omega_{W}^{-1}(s)=1-\frac{g^{2}(s)}{s}\left\{\frac{p_{W}}{g^{2}\left(p_{W}\right)}-\frac{1}{16 \pi^{2}}\left[f_{W}(s)-f_{W}\left(p_{W}\right)\right]\right\}, \\
& \omega_{Z}^{-1}(s)=1-\frac{g^{2}(s)}{c^{2}(s) s}\left\{\frac{c^{2}\left(p_{Z}\right)}{g^{2}\left(p_{Z}\right)} p_{Z}-\frac{1}{16 \pi^{2}}\left[f_{Z}(s)-f_{Z}\left(p_{W}\right)\right]\right\} .
\end{aligned}
$$

The explicit form of the $f_{W, Z}$-functions is given in [9]. The complete one-loop re-summation in the 't Hooft-Feynman gauge is equivalent to some effective unitary-gauge $W$-propagator. The whole amplitude can be written in terms of a $W$-boson exchange diagram, if we make use of the following effective propagator:

$$
\Delta_{\mathrm{eff}}^{\mu \nu}=\frac{1}{p^{2}+M^{2}-S_{W}^{0}}\left[\delta^{\mu \nu}+\frac{p^{\mu} p^{\nu}}{M^{2}\left(p^{2}\right)}\right] .
$$

The explicit form of $M^{2}\left(p^{2}\right)$ is, again given in [9]. We now define the following line-shape functions:

$$
L_{\mathrm{FL}}(s)=\frac{s^{2}}{\left(s-\operatorname{Re} p_{W}\right)^{2}+\left(\operatorname{Im} p_{W}\right)^{2}} \frac{1}{|R(s)|^{2}},
$$



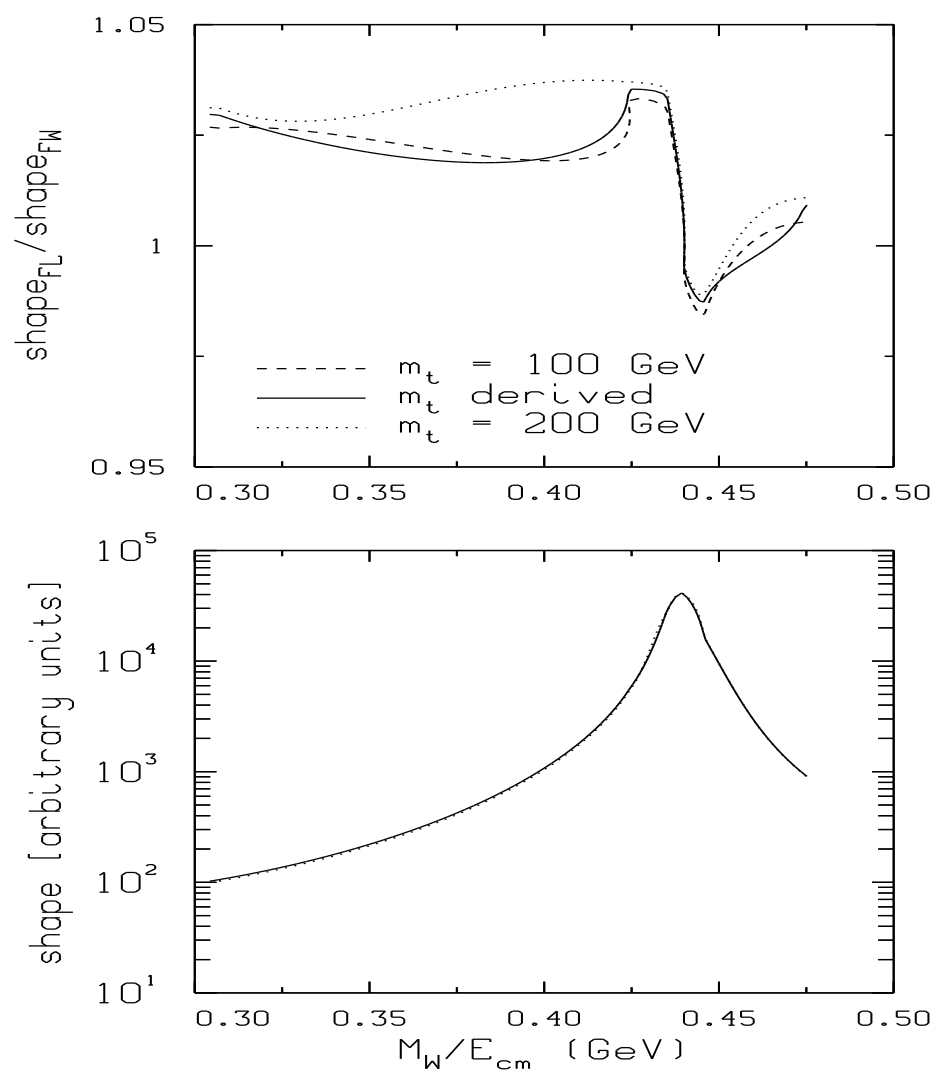

Figure 2: Comparison between the Fixed-Width and the Fermion-Loop line-shapes, Eq.(14).

$$
L_{\mathrm{FW}}(s)=\frac{s^{2}}{\left(s-M_{W}^{2}\right)^{2}+M_{W}^{2} \Gamma_{W}^{2}} .
$$

with a running $\rho$-factor

$$
R(s)=1+\frac{g^{2}(s)}{16 \pi^{2}} \frac{f_{W}(s)-f_{W}\left(p_{W}\right)+p_{W}\left[\Pi_{3 Q}\left(p_{W}\right)-\Pi_{3 Q}(s)\right]}{s-p_{W}} .
$$

Here $M_{W}, \Gamma_{W}$ are the on-shell $W$ mass and total width, respectively. The two line-shapes are compared in Fig. 2 for different values of the top quark mass. We are using complex-mass renormalization but we only include fermionic corrections. Therefore, we can start with the Fermi coupling constant but also with $M_{W}$ as an input parameter. Equating the corresponding renormalization conditions yields a relation between $M_{Z}, G_{F}, \operatorname{Re}\left\{\alpha\left(M_{Z}^{2}\right)^{-1}\right\}, M_{W}$, and $m_{t}$, see [7]. This relation can be solved iteratively for $m_{t}$. For the following input parameter set,

$$
M_{W}=80.350 \mathrm{GeV}, \quad M_{Z}=91.1867 \mathrm{GeV}, \quad G_{F}=1.16639 \times 10^{-5} \mathrm{GeV}^{-2},
$$

we obtain the following solution:

$$
\mu_{W}=\sqrt{\operatorname{Re}\left(p_{W}\right)}=80.324 \mathrm{GeV}, \quad \gamma_{W}=-\frac{\operatorname{Im}\left(p_{W}\right)}{\mu_{W}}=2.0581 \mathrm{GeV}, \quad m_{t}=148.62 \mathrm{GeV} .
$$




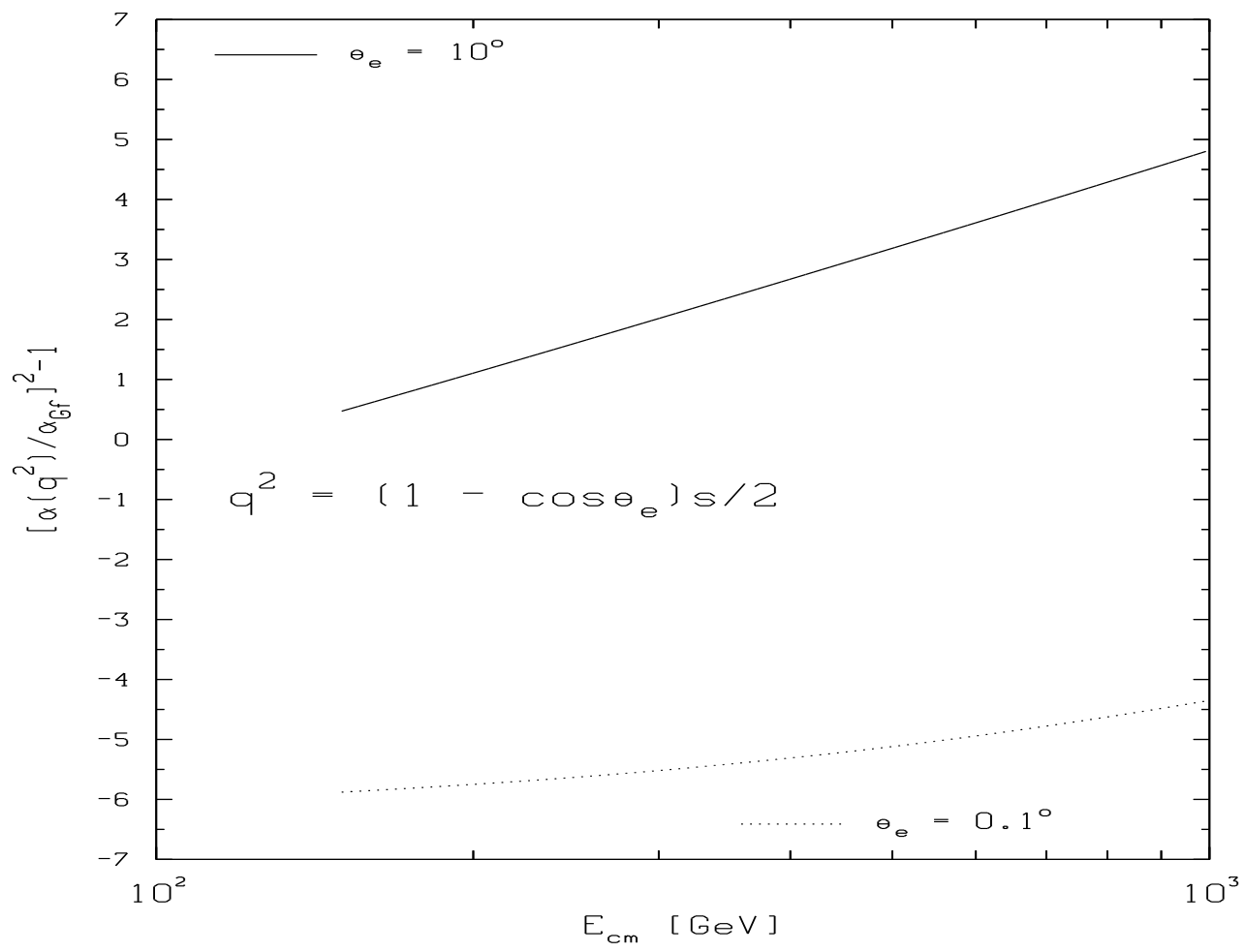

Figure 3: Running of $\alpha_{\mathrm{QED}}\left(q^{2}\right)$ in the $t$-channel.

The $26 \mathrm{MeV}$ difference between $M_{W}$ and $\mu_{W}$ is responsible for the sharp transition around $80 \mathrm{GeV}$ that can be seen in Fig. 2. Apart from that, the Fermion-Loop line-shape can be few percents above the Fixed-Width line shape. The behavior of $e^{2}\left(q^{2}\right)$ in the $t$-channel and, to some extent, the ratio between Fermion-Loop and Fixed-Width cross-sections is crucially dependent on which regime we are considering. A careful examination of Fig. 3 shows the following: if we choose

$$
<q^{2}>=\left(1-<\cos \theta_{e}>\right) \frac{s}{2},
$$

and use $10^{\circ}$ or $0.1^{\circ}$ as representative for LACC20 or SACC20, we obtain a ratio

$$
\begin{gathered}
{\left[\frac{\alpha\left(q^{2}\right)}{\alpha_{G_{F}}}\right]^{2}-1 \rightarrow-6 \% \div-4 \%, \quad<\theta_{e}>=0.1^{\circ}} \\
{\left[\frac{\alpha\left(q^{2}\right)}{\alpha_{G_{F}}}\right]^{2}-1 \rightarrow+0.5 \% \div+5 \%, \quad<\theta_{e}>=10^{\circ}}
\end{gathered}
$$

for $\sqrt{s}$ ranging from $150 \mathrm{GeV}$ to $1 \mathrm{TeV}$. Therefore, the effect of a running $\alpha_{\mathrm{QED}}$ in the $t$-channel is to decrease/increase the cross-section, with respect to the Fixed-Width scheme, for SACC20/LACC20.

A final ingredient, existing in the Fermion-Loop scheme, is represented by the inclusion of vertices that are $m_{t}$-dependent. The top-quark contributions are particularly important for delayed-unitarity effects. In this respect also terms involving the totally-antisymmetric $\varepsilon$-tensor (originating from vertex corrections) are relevant. While such terms are absent for complete generations of massless fermions owing to the anomaly cancellations, they show up for finite fermion masses. 
All details concerning vertices can be found in [9] where one can find the explicit expressions for the vertex form-factors in terms of standard $C$-functions $[16$.

\section{The diagrams.}

As already mentioned, there are 20 Feynman diagrams that contribute for $e \bar{\nu}_{e} u \bar{d}$ final states, and 56 for $e^{+} e^{-} \nu_{e} \bar{\nu}_{e}$. They are well known to the expert working in the field, but we present them for convenience of the less specialized reader. For simplicity, we only consider the CC20 family. The most familiar part of CC20 is the CC03 one, with three diagrams as depicted in Fig. \&, The next set of

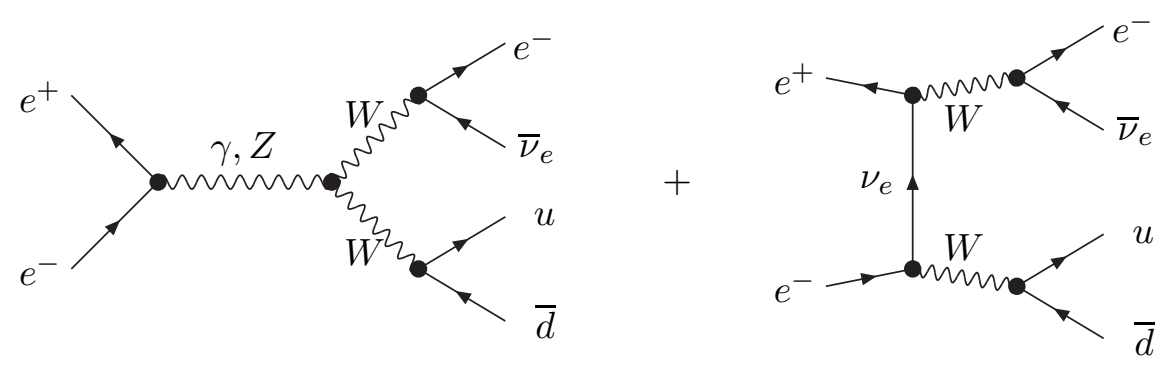

Figure 4: The CC03 family of diagrams, annihilation $\oplus$ conversion.

diagrams needed to complete the $s$-channel component shows up with the CC11 class where we add to the annihilation and conversion CC03 diagrams all topologies corresponding to pair production of fermions. The CC11 diagrams not in CC03 are single-resonant and they are shown in Fig. 5 Finally, we
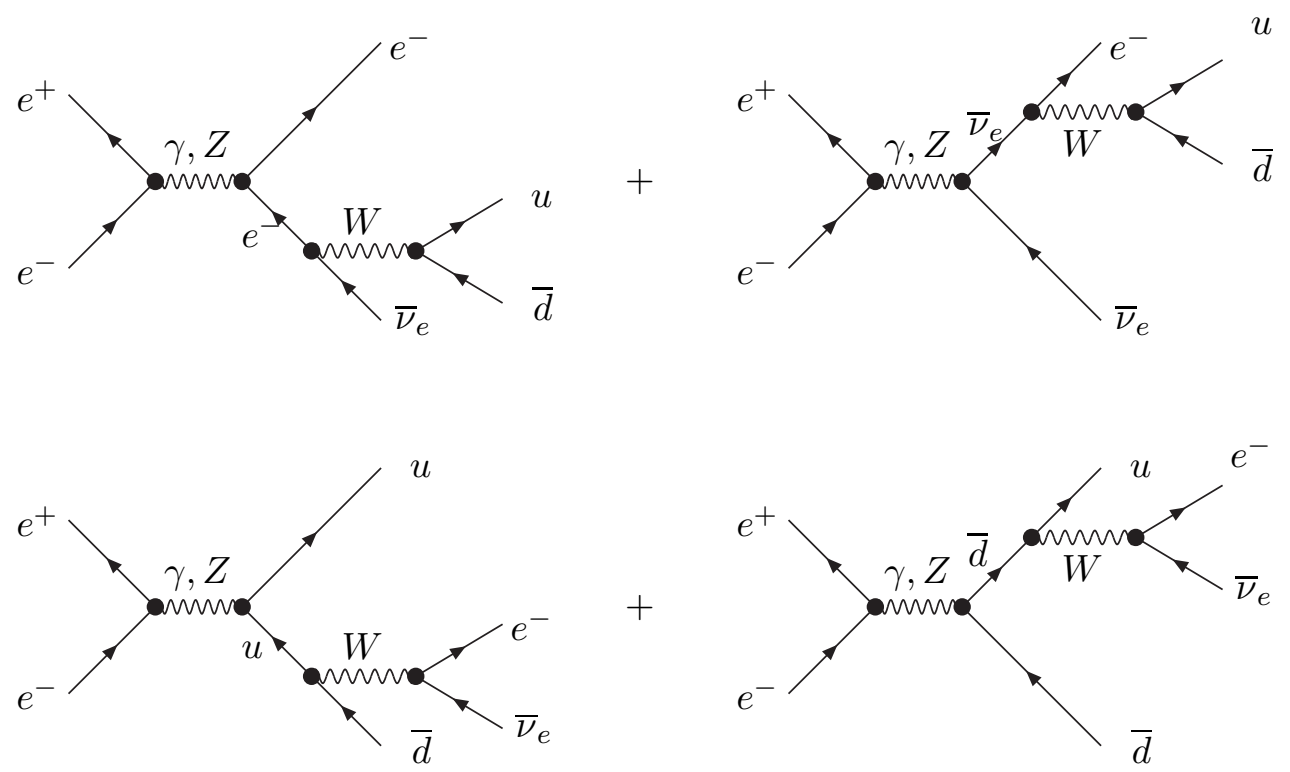

Figure 5: Diagrams belonging to the CC11 - CC03 family.

have the 10 diagrams forming the $t$-channel part. In the latter part, one diagram has a $W$-exchange, 
five a $Z$-exchange and four a $\gamma$-exchange. As we have shown in [9], this picture is not changed by the inclusion of one-loop fermionic corrections.

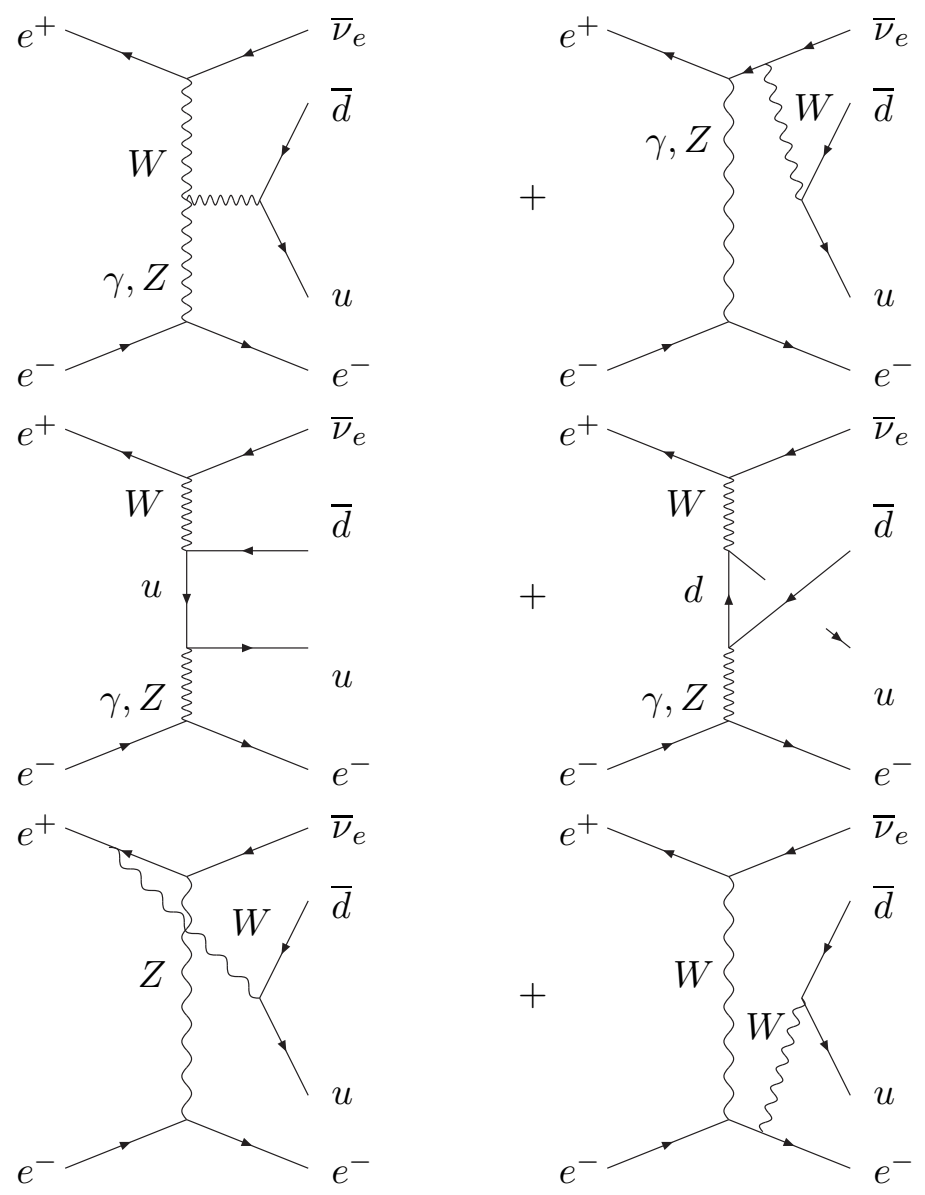

Figure 6: The $t$-channel component of the CC20 family of diagrams: fusion, bremsstrahlung and multi-peripheral.

\section{Implementing the Fermion-Loop scheme.}

Here we follow closely the spirit of Sect. 6 of [9]: once gauge invariance is preserved then we are allowed to investigate the numerical relevance of masses and to neglect some, if convenient. For the photon $t$-channel diagrams, the amplitude squared, summed over spin and integrated over the phase space of $\nu_{e} u \bar{d}$, can be written as follows:

$$
\begin{aligned}
\frac{1}{4} L_{\mu \nu}^{\gamma \gamma} W_{\gamma \gamma}^{\mu \nu} & =2 \frac{m_{e}^{2}}{(X y s)^{2}} W_{\gamma \gamma}^{1}-\frac{1}{X y s} W_{\gamma \gamma}^{1} \\
& +\frac{2}{X y^{2} s}\left(1-\frac{1}{y}\right) W_{\gamma \gamma}^{2}+\frac{2}{y^{2} s}\left[\frac{1}{(X+1)^{2}}+\frac{1}{X+1}\right]\left(\frac{1}{y}+1\right) W_{\gamma \gamma}^{2} \\
& +\frac{1}{y s} \frac{1}{X+1} W_{\gamma \gamma}^{2}
\end{aligned}
$$


In Eq.(20) $L$ is the usual leptonic tensor while $W$ is a CC20 tensor, pertaining to the specific process under consideration. U(1) gauge invariance, proven in [9], allows for the following decomposition of $W_{\gamma \gamma}^{\mu \nu}$,

$$
\begin{aligned}
W_{\mu \nu}^{\gamma \gamma} & =W_{\gamma \gamma}^{1}\left[-\delta_{\mu \nu}+\frac{Q_{-\mu} Q_{\nu}}{Q_{-}^{2}}\right]-W_{\gamma \gamma}^{2} \frac{Q_{-}^{2}}{\left(p_{+} \cdot Q_{-}\right)^{2}}\left(p_{+\mu}-\frac{p_{+} \cdot Q_{-}}{Q_{-}^{2}} Q_{-\mu}\right) \\
& \times\left(p_{+\nu}-\frac{p_{+} \cdot Q_{-}}{Q_{-}^{2}} Q_{-\nu}\right) .
\end{aligned}
$$

Moreover, with momenta assignment $e^{+}\left(p_{+}\right) e^{-}\left(p_{-}\right) \rightarrow e^{-}\left(q_{-}\right) \bar{\nu}_{e}\left(q_{+}\right) u(k) \bar{d}(\bar{k}), Q_{-}=p_{-} q_{-}$, we have introduced the variable $y$, equivalent to the fraction of the electron energy carried by the photon, and the variable $X$ according to the following definitions:

$$
\begin{aligned}
p_{+} \cdot Q_{-} & =\frac{1}{2}\left[m_{e}^{2}-(X+1) y s\right] \\
Q_{-}^{2} & =X y s, \quad\left(p_{+}+p_{-}\right)^{2}=-s .
\end{aligned}
$$

Note that our approach is akin to the Weizsäcker-Williams approximation. However, we have gone beyond this approximation where one replaces $W_{\gamma \gamma}^{1,2}\left(Q_{-}^{2}, y\right)$ of Eq.(21) with $W_{\gamma \gamma}^{1,2}(0, y)$. In our approach, we retain the full $Q_{-}^{2}$-dependence of the photonic exchange in the $t$-channel, CC20, diagrams. The sole approximation that we have used is to retain only those parts that, after integration, produce logarithmically enhanced terms, $\mathcal{O}\left(\ln \left(m_{e}^{2} / s\right)\right)$, and constant terms, $\mathcal{O}(1)$, therefore neglecting all terms proportional to $\mathcal{O}\left(\left(m_{e}^{2} / s\right)^{n}\right)$. Since $\min \left\{Q_{-}^{2}\right\} \sim m_{e}^{2}$ this procedure is equivalent to say that we keep, prior to the integration over $Q_{-}^{2}, y$, all terms that are of order

$$
\mathcal{O}\left(\frac{m_{e}^{4}}{Q_{-}^{6}}\right), \quad \mathcal{O}\left(\frac{m_{e}^{2}}{Q_{-}^{4}}\right), \quad \mathcal{O}\left(\frac{1}{Q_{-}^{2}}\right), \quad \mathcal{O}(1) .
$$

By inspection, it is easily seen that the $\gamma-Z, Z-Z, W-W$ etc parts of the $t$-channel and the full $s$-channel component only contain terms of $\mathcal{O}(1)$. Therefore, for the non-photonic part of the $t$-channel, for its interference with the photonic part and for the $s$-channel we can use the old version of WTO, employing the massless Fermion-Loop scheme. For the photonic part of the $t$-channel we have introduced in the latest version of WTO the massive Fermion-Loop scheme as described in [9].

In contrast with a previous calculation [1], performed with the program wTO in the Fixed-Width scheme, here we do not have a problem of introducing a small cone around the beam axis matching the internal region with then external one. Indeed, as noted before, we have been able to avoid the Weizsäcker-Williams approximation. In other words, the evaluation of the $t$-channel component is now exact in the whole range of the photon momentum.

One should not get the wrong impression that our approximation, massive for the photonic part of the $t$-channel and massless for the rest, is an inconsistent one. Therefore, it is useful to explain the procedure in more detail. We construct gauge invariant subsets of amplitudes, consider their gauge invariant squares and interferences and, after that, we consistently neglect all terms of $\mathcal{O}\left(\mathrm{m}_{f}^{2} / \mathrm{s}\right)$.

The grand total of 20 Feynman diagrams is, first of all, split into a $10 \mathrm{~s}$-channel part and a 10 $t$-channel part. In the latter part, one diagram has a $W$-exchange, five a $Z$-exchange and four a $\gamma$-exchange. As we have shown, this picture is not changed by the inclusion of one-loop fermionic 
corrections: when all transitions are properly taken into account, we still end up with the $1-5-4$ subdivision described above, as long as the fermion-anti-fermion-vector-boson couplings are described in terms of the re-summed expressions.

This $s \oplus t$ splitting is a gauge invariant one, but we can further characterize additional set of diagrams. The argument is as follows: Take $e^{+} \mu^{-} \rightarrow \bar{\nu}_{e} \mu^{-} u \bar{d}$. Only the CC20 $t$-channel diagrams contribute and, since these are all diagrams that we need, this set is gauge invariant. Next, take $e^{+} \nu_{\mu} \rightarrow \bar{\nu}_{e} \nu_{\mu} u \bar{d}$. It is made, again, only with $t$-channel parts but the photon does not contribute, so that we have $10-4=6$ diagrams. Moreover, if one writes any $Z$ current as $J=J_{Q}+J_{L}$ (with $J_{L}$ proportional to $\gamma_{+}$), only $J_{L}$ contributes here, because of the neutrinos, so that we have five $J_{L}^{Z}$ diagrams plus one $W$ diagram that form a gauge invariant set. Since the whole $t$-channel is gauge invariant, the eight diagrams, four with photons and four with $J_{Q}^{Z}$, must form a gauge invariant subset. This remains true for one-loop corrections when writing everything in terms of running objects and including vertices.

Consider, now, the eight $J^{\gamma} \oplus J_{Q}^{Z}, t$-channel diagrams. They can be decomposed in a product $L_{\mu \nu} W^{\mu \nu}$. For the $W$-tensors we obtain

$$
\begin{aligned}
W_{\mu \nu}^{\gamma \gamma} & =W_{\gamma \gamma}^{1}\left[-\delta_{\mu \nu}+\frac{Q_{-\mu} Q_{\nu}}{Q_{-}^{2}}\right]-W_{\gamma \gamma}^{2} \frac{Q_{-}^{2}}{\left(p_{+} \cdot Q_{-}\right)^{2}}\left(p_{+\mu}-\frac{p_{+} \cdot Q_{-}}{Q_{-}^{2}} Q_{-\mu}\right) \\
& \times\left(p_{+\nu}-\frac{p_{+} \cdot Q_{-}}{Q_{-}^{2}} Q_{-\nu}\right), \\
W_{\mu \nu}^{\gamma Z} & =W_{\gamma Z}^{1}\left[-\delta_{\mu \nu}+\frac{Q_{-\mu} Q_{\nu}}{Q_{-}^{2}}\right]-W_{\gamma Z}^{2} \frac{Q_{-}^{2}}{\left(p_{+} \cdot Q_{-}\right)^{2}}\left(p_{+\mu}-\frac{p_{+} \cdot Q_{-}}{Q_{-}^{2}} Q_{-\mu}\right) \\
& \times\left(p_{+\nu}-\frac{p_{+} \cdot Q_{-}}{Q_{-}^{2}} Q_{-\nu}\right) \\
& +\frac{W_{\gamma Z}^{3}}{p_{+} \cdot Q_{-}}\left(\mu, \nu, Q_{-}, p_{+}\right)+\frac{W_{\gamma Z}^{4}}{p_{+} \cdot Q_{-}} p_{+\mu} p_{+\nu} \\
& +\frac{W_{\gamma Z}^{5}}{p_{+} \cdot Q_{-}}\left(p_{+\mu} Q_{-\nu}+p_{+\nu} Q_{-\mu}\right)+W_{\gamma Z}^{6} \frac{Q_{-\mu} Q_{-\nu}}{Q_{-}^{2}} . \\
W_{\mu \nu}^{Z Z} & =W_{Z Z}^{1}\left[-\delta_{\mu \nu}+\frac{Q_{-\mu} Q_{\nu}}{Q_{-}^{2}}\right]-W_{Z Z}^{2} \frac{Q_{-}^{2}}{\left(p_{+} \cdot Q_{-}\right)^{2}}\left(p_{+\mu}-\frac{p_{+} \cdot Q_{-}}{Q_{-}^{2}} Q_{-\mu}\right) \\
& \times\left(p_{+\nu}-\frac{p_{+} \cdot Q_{-}}{Q_{-}^{2}} Q_{-\nu}\right)+\frac{W_{Z Z}^{3}}{p_{+} \cdot Q_{-}}\left(\mu, \nu, Q_{-}, p_{+}\right) \\
& +\frac{W_{Z Z}^{4}}{p_{+} \cdot Q_{-}} p_{+\mu} p_{+\nu}+\frac{W_{Z Z}^{5}}{p_{+} \cdot Q_{-}}\left(p_{+\mu} Q_{-\nu}+p_{+\nu} Q_{-\mu}\right)+W_{Z Z}^{6} \frac{Q_{-\mu} Q_{-\nu}}{Q_{-}^{2}} .
\end{aligned}
$$

For $J_{Q}^{Z}$ the $W^{3}$ form factor does not contribute in the $L \otimes W$ contraction.

Strictly speaking, only $J_{\mu}^{\gamma}$ satisfies the condition $Q_{-}^{\mu} J_{\mu}^{\gamma}=0$. However, by using Ward identities, one can easily prove that $Q_{-}^{\mu} J_{\mu}^{Z}=\mathcal{O}\left(m_{f}\right)$, owing to a direct coupling of the $\phi^{0}$ with fermions and to the absence of a $W^{+} W^{-} \phi^{0}$ tree-level coupling.

Let's repeat that, inside a gauge invariant subset of diagrams we only keep the terms shown in 
Eq.(23).

Let's start with $L^{\mu \nu} W_{\mu \nu}^{\gamma \gamma}$. The result is given in Eq.(20) and, according to our strategy of neglecting terms of $\mathcal{O}\left(m_{f}^{2} / s\right)$, we can compute the $W_{\gamma \gamma}$-tensor in the massless approximation.

The most delicate case seems to be the interference, $\gamma \otimes Z$. Since, schematically, $W_{\mu \nu}=J_{\mu}^{\dagger} J_{\nu}$, this interference can be written as

$$
W_{\mu \nu}^{\gamma Z}=\left(J_{\mu}^{\gamma}\right)^{\dagger} J_{\nu}^{Z}+\left(J_{\mu}^{Z}\right)^{\dagger} J_{\nu}^{\gamma}
$$

Multiplication by $Q_{-}^{\mu}$ or by $Q_{-}^{\nu}$ gives terms of $\mathcal{O}\left(m_{f}\right)$ since the e.m. current is conserved. Therefore, the form factors $W_{\gamma Z}^{4,5,6}$ are of $\mathcal{O}\left(m_{f}\right)$. Furthermore, we see from Eq.(25) that $Q_{-}^{\mu} Q_{-}^{\nu} W_{\mu \nu}^{\gamma Z}=0$. It follows:

$$
\begin{aligned}
\mathcal{W}_{\mu \nu}^{\gamma Z} & =\frac{W_{\gamma Z}^{4}}{p_{+} \cdot Q_{-}} p_{+\mu} p_{+\nu}+\frac{W_{\gamma Z}^{5}}{p_{+} \cdot Q_{-}}\left(p_{+\mu} Q_{-\nu}+p_{+\nu} Q_{-\mu}\right)+W_{\gamma Z}^{6} \frac{Q_{-\mu} Q_{-\nu}}{Q_{-}^{2}}, \\
Q_{-}^{\nu} \mathcal{W}_{\mu \nu}^{\gamma Z} & =J_{\phi}^{p} p_{+\mu}+J_{\phi}^{q} Q_{-\mu},
\end{aligned}
$$

where the $J_{\phi}^{p, q}$ form factors are related to the same set of diagrams with the replacement $Z \rightarrow \phi^{0}$ and they are $\mathcal{O}\left(m_{f}\right)$. Multiplication by $Q_{-}^{\mu}$ gives zero, with solution

$$
\begin{aligned}
J_{\phi}^{p} & =-\frac{Q_{-}^{2}}{p_{+} \cdot Q_{-}} J_{\phi}^{q}, \\
W_{\gamma Z}^{6} & =1, \quad W_{\gamma Z}^{5}=J_{\phi}^{q}-1, \quad W_{\gamma Z}^{4}=\frac{Q_{-}^{2}}{p_{+} \cdot Q_{-}}\left(1-2 J_{\phi}^{q}\right) .
\end{aligned}
$$

In this particular case, the $L \otimes W$ contraction gives the following result:

$$
\begin{aligned}
\frac{1}{4} L_{\mu \nu}^{\gamma Z} W_{\gamma Z}^{\mu \nu} & =s_{\theta}^{2}\left[-4 W_{\gamma Z}^{1}+\frac{8}{y(X+1)^{2}}\left(1-\frac{1}{y}\right) W_{\gamma Z}^{2}\right. \\
& +4\left(1-\frac{1}{X+1}\right) W_{\gamma Z}^{2}+\frac{4}{y X}\left(1-\frac{1}{y}\right) W_{\gamma Z}^{4}+\frac{4}{y(X+1)}\left(\frac{1}{y}-1\right) W_{\gamma Z}^{4} \\
& \left.+2 \frac{X}{X+1}\left(W_{\gamma Z}^{5}+W_{\gamma Z}^{6}\right)\right] .
\end{aligned}
$$

The only term which is proportional to $1 / X$ multiplies $W_{\gamma z}^{4}$. Note, however, that this form factor is proportional to $\mathrm{X}$ by virtue of Eq.27). Therefore, the contraction does not contain any term that is divergent in the massless limit and we can neglect fermion masses.

The $Z Z$ terms do not have any divergence to start with so that, in our procedure, we can neglect all fermion masses. The same is true for the subset formed by the $J_{L}^{Z}$ diagrams plus one $W$ diagram, for the $s$-channel diagrams, for their mutual interferences and for their interference with the photonic $t$-channel part. The latter follows from the same line of argument used in studying the $J^{\gamma}-J_{Q}^{Z}$ interference and by recalling that a cross-section is a sum of cut diagrams: infrared power counting shows that there are no mass singular contributions in the interferences. As far as the $s \otimes t$ interference is concerned this confirms previous numerical findings, see Fig. 4 of the first reference in [2]. 


\section{Numerical results.}

In this paper we will consider two examples belonging to the single- $W$ family:

1. $e^{+} e^{-} \rightarrow u \bar{d} e \nu,\left|\cos \theta_{e}\right|>0.997, M(u \bar{d})>45 \mathrm{GeV}$;

2. $e^{+} e^{-} \rightarrow e \nu \mu \nu(\gamma),\left|\cos \theta_{e}\right|>0.997, E_{\mu}>15 \mathrm{GeV},\left|\cos \theta_{\mu}\right|<0.95$.

Our Input Parameter Set is specified by

$$
\sqrt{s}=183 / 189 / 200 \mathrm{GeV}, \quad M_{W}=80.350 \mathrm{GeV}, \quad M_{Z}=91.1867 \mathrm{GeV},
$$

Moreover, we are mostly interested in the variations induced by the Fermion-Loop scheme with respect to the Fixed-Width scheme. Therefore, we will not include other effects that are nevertheless needed in any realistic evaluation of observables:

1. QED Initial State Radiation;

2. QCD naive corrections;

3. Final State Coulomb correction factor;

4. Final State QED radiation

5. Anomalous couplings.

Furthermore, we use trivial CKM matrix element, lepton masses according to PDG [17], light quark masses $5 \mathrm{MeV}$ (up) and $10 \mathrm{MeV}$ (down), $G_{F}=1.1663710^{-5}$ (recent Fermi coupling).

When working in the Fixed-Width approach we use the $G_{F}$-scheme, that is

$$
s_{\theta}^{2}=1-\frac{M_{W}^{2}}{M_{Z}^{2}}, \quad \alpha \equiv \alpha_{G_{F}}=4 \sqrt{2} \frac{G_{F} M_{W}^{2} s_{\theta}^{2}}{4 \pi}
$$

Numerical (integration) errors returned for the cross-section computed with $0<\theta_{e}<\theta_{e}^{\max }$ and $\theta_{e}^{\max }=0.1^{\circ} \div 0.4^{\circ}$ are, typically, of order $0.05 \div 0.1 \%$ for $u \bar{d}$ and $\nu_{\mu} \mu^{+}$final states, respectively. We start in Tab.(11) by comparing FL and FW cross-sections at $\sqrt{s}=183 \mathrm{GeV}$. Clearly, the bulk of the cross-section is for very small scattering angle of the electron, dominated by the first bin. The differences between FL-scheme and FW-scheme are decreasing when $\theta_{e}^{\max }$ becomes larger and, from the shape of the angular distribution, we infer smaller differences in the higher bins.

To continue our discussion of the numerical results we stay at $\sqrt{s}=183 \mathrm{GeV}$ and consider now the angular distribution, $d \sigma / d \theta_{e}$ for the $u \bar{d} e^{-} \bar{\nu}_{e}$ final states. The results are shown in Fig. 7 . From Fig. 7 we see that the FL prediction is lower than the $\mathrm{FW}$ one, from $-7.46 \%$ in the bin $0^{\circ}-0.1^{\circ}$ to $-5.56 \%$ in the bin $0.3^{\circ}-0.4^{\circ}$. Correspondingly, the first bin is 6.78 higher than the second one, $11.60(16.37)$ than the third(fourth) one. This is not a surprise, since the first bin represents $50 \%$ of the total single- $W$ cross-section.

Always in the same figure, we have reported the behavior of $\left[\alpha\left(q^{2}\right) / \alpha_{G_{F}}-1\right]^{2}$ as a function of $\theta_{e}$ for three values of $y$, Eq.(22), using the appropriate relation: $q^{2}=q^{2}\left(\theta_{e}, y\right)$. The behavior of FL/FW-1, when we vary $\theta_{e}$, is very similar to the one given by the ratio of coupling constants, indicating that the bulk of the effect is in the running of the e.m. coupling constant. 


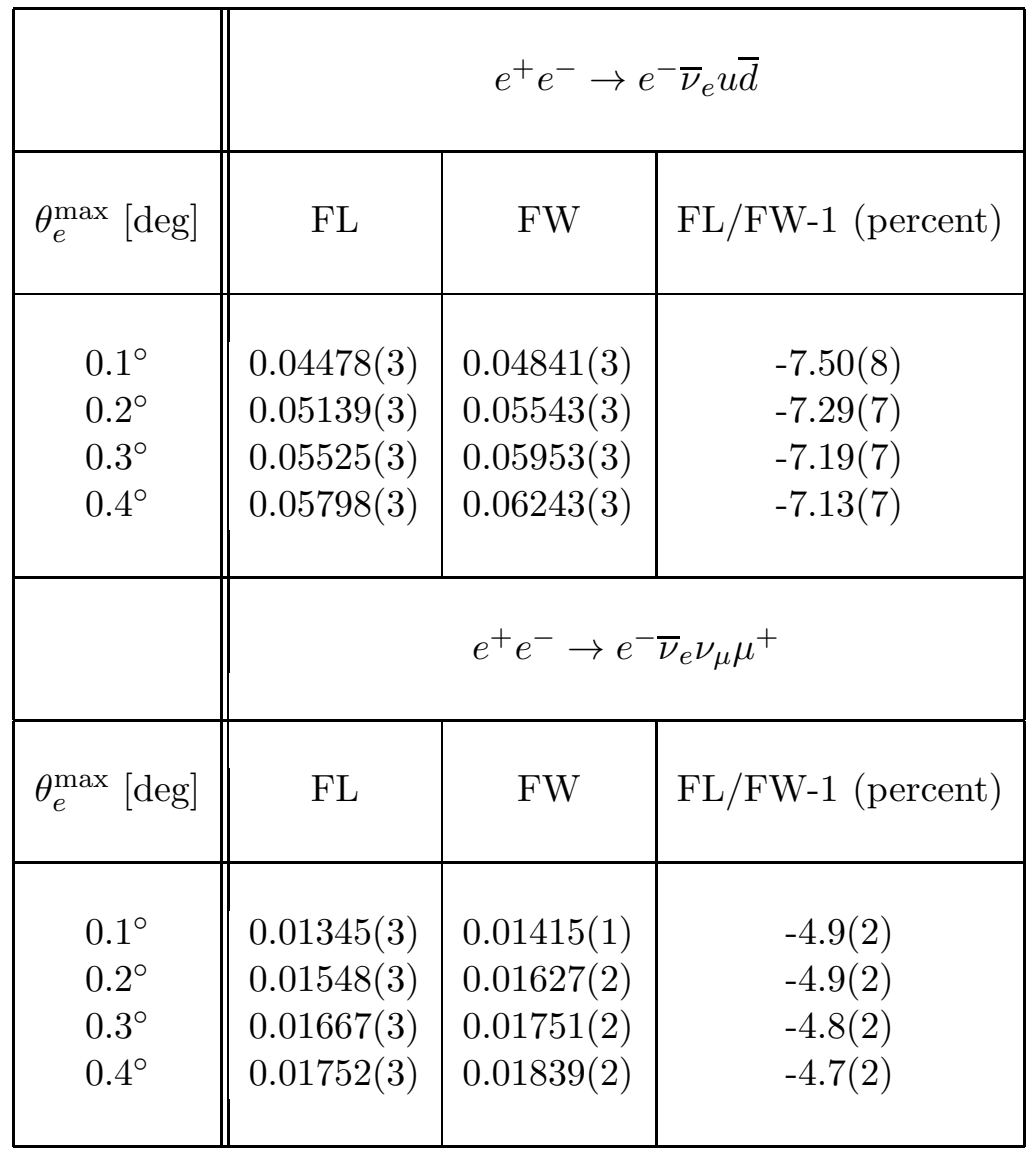

Table 1: $\quad \sigma$ in pb for the processes $e^{+} e^{-} \rightarrow e^{-} \bar{\nu}_{e} u \bar{d}\left(\nu_{\mu} \mu^{+}\right)$, for $\sqrt{s}=183 \mathrm{GeV}$ and for $\theta_{e}<\theta_{e}^{\max }$. Kinematical cuts are as described in the main text. The number in parenthesis shows the statistical error of the numerical integration on the last digit. 


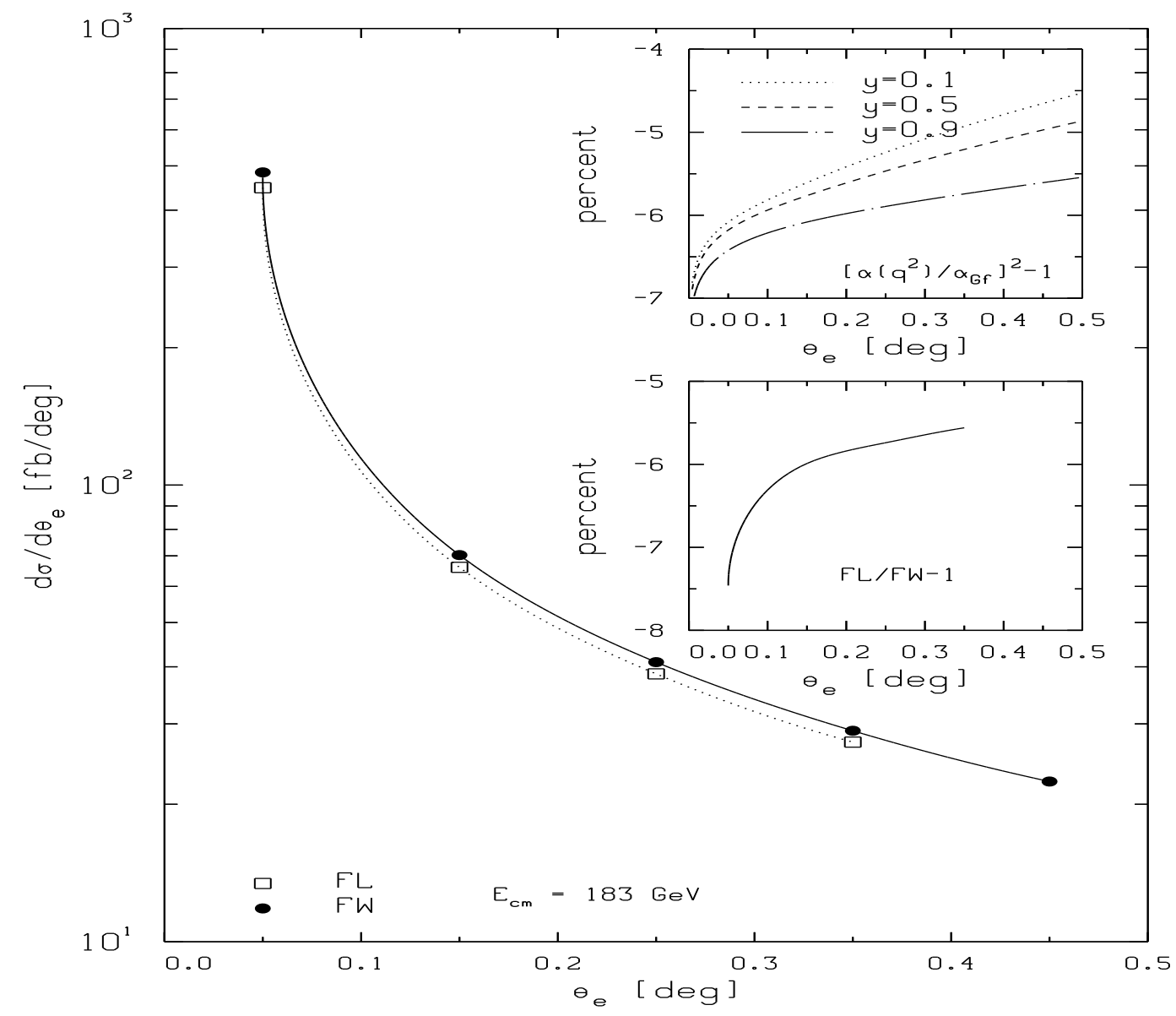

Figure 7: $d \sigma / d \cos \theta_{e}\left[\mathrm{fb} /\right.$ degrees] for $e^{+} e^{-} \rightarrow u \bar{d} e^{-} \bar{\nu}_{e}$ with $M(u \bar{d})>45 \mathrm{GeV}$ and $\sqrt{s}=183 \mathrm{GeV}$.

We have repeated the calculation for all the three energies, finding similar results that are shown in Fig. 8. For completeness we have reported the numerical results for the three energies in Tab.(2), where the first entry is Fixed-Width distribution and the second entry is Fermion-Loop one. Only the first four bins are shown, owing to the fact that they are the most significant in the distribution. The third entry in Tab.(2) gives FL/FW-1 in percent. After discussing the angular distribution we consider the total - single- $W$ - cross-section, defined by an acceptance cut of $\left|\cos \theta_{e}\right|>0.997$, again for the high-mass case, i.e. $M(u \bar{d})>45 \mathrm{GeV}$. It is reported in Tab.(3)), where one sees that differences between schemes are of the order of $5 \%$ for all energies, supporting the use of some effective $\alpha\left(<q^{2}>\right)$ with $\left\langle q^{2}\right\rangle=\left(1-\cos \left\langle\theta_{e}>\right) s / 2\right.$ where $\left\langle\theta_{e}>\approx 0.1^{\circ} \div 0.2^{\circ}\right.$.

We have also computed the single- $W$ cross-sections for a fixed mass of the top quark, $m_{t}=$ $173.8 \mathrm{GeV}$, without finding any significative difference with the previous case where $m_{t}$ is fixed by a consistency relation: they give $83.29(6) \mathrm{fb}, 93.80(7) \mathrm{fb}$ and $113.68(8) \mathrm{fb}$ for $\sqrt{s}=183 \mathrm{GeV}, 189 \mathrm{GeV}$ and $200 \mathrm{GeV}$.

Next we consider $e^{+} e^{-} \rightarrow e \nu \mu \nu$, with $\left|\cos \theta_{e}\right|>0.997, E_{\mu}>15 \mathrm{GeV}$, and $\left|\cos \theta_{\mu}\right|<0.95$. The angular cut on the muon direction is chosen to prevent the logarithmic enhancement that would, otherwise, show up in the multi-peripheral terms. Indeed, when the electron is lost in the beam pipe, and the quasi-massless fermion is emitted parallel to the (quasi-real) photon then the internal fermion propagator will produce an enhancement in the cross section. In Tab.(四) we report the comparison 


\begin{tabular}{|c||c|c|c|}
\hline$\theta_{e}[\mathrm{Deg}]$ & $\sqrt{s}=183 \mathrm{GeV}$ & $\sqrt{s}=189 \mathrm{GeV}$ & $\sqrt{s}=200 \mathrm{GeV}$ \\
\hline \multirow{3}{*}{$0.0^{\circ} \div 0.1^{\circ}$} & 0.48395 & 0.54721 & 0.67147 \\
& 0.44784 & 0.50695 & 0.62357 \\
& -7.46 & -7.36 & -7.13 \\
& & & \\
\hline \multirow{2}{*}{$0.1^{\circ} \div 0.2^{\circ}$} & 0.07026 & 0.07815 & 0.09323 \\
& 0.06605 & 0.07357 & 0.08798 \\
& -5.99 & -5.86 & -5.63 \\
& & & \\
\hline \multirow{3}{*}{$0.2^{\circ} \div 0.3^{\circ}$} & 0.04095 & 0.04554 & 0.05433 \\
& 0.03860 & 0.04298 & 0.05141 \\
& -5.74 & -5.62 & -5.37 \\
& & & \\
& & & \\
& & & \\
$0.3^{\circ} \div 0.4^{\circ}$ & 0.02897 & 0.03223 & 0.03845 \\
& 0.02736 & 0.03045 & 0.03646 \\
& -5.56 & -5.52 & -5.18 \\
& & & \\
\hline
\end{tabular}

Table 2: $d \sigma / d \theta_{e}$ in [pb/degrees] for the process $e^{+} e^{-} \rightarrow e^{-} \bar{\nu}_{e} u \bar{d}$, for $M(u \bar{d})>45 \mathrm{GeV}$. First entry is Fixed-Width distribution, second entry is Fermion-Loop one and third entry if FL/FW-1 in percent.

\begin{tabular}{|c||c|c|c|}
\hline$\sqrt{s}$ & FW & FL & FL/FW-1 (percent) \\
\hline $183 \mathrm{GeV}$ & $88.17(44)$ & $83.28(6)$ & $-5.5(5)$ \\
$189 \mathrm{GeV}$ & $98.45(25)$ & $93.79(7)$ & $-4.7(3)$ \\
$200 \mathrm{GeV}$ & $119.77(67)$ & $113.67(8)$ & $-5.1(5)$ \\
\hline
\end{tabular}

Table 3: Total single- $W$ cross-section in fb for the process $e^{+} e^{-} \rightarrow e^{-} \bar{\nu}_{e} u \bar{d}$, for $M(u \bar{d})>45 \mathrm{GeV}$ and $\left|\cos \theta_{e}\right|>0.997$. The number in parenthesis shows the statistical error of the numerical integration on the last digit. 


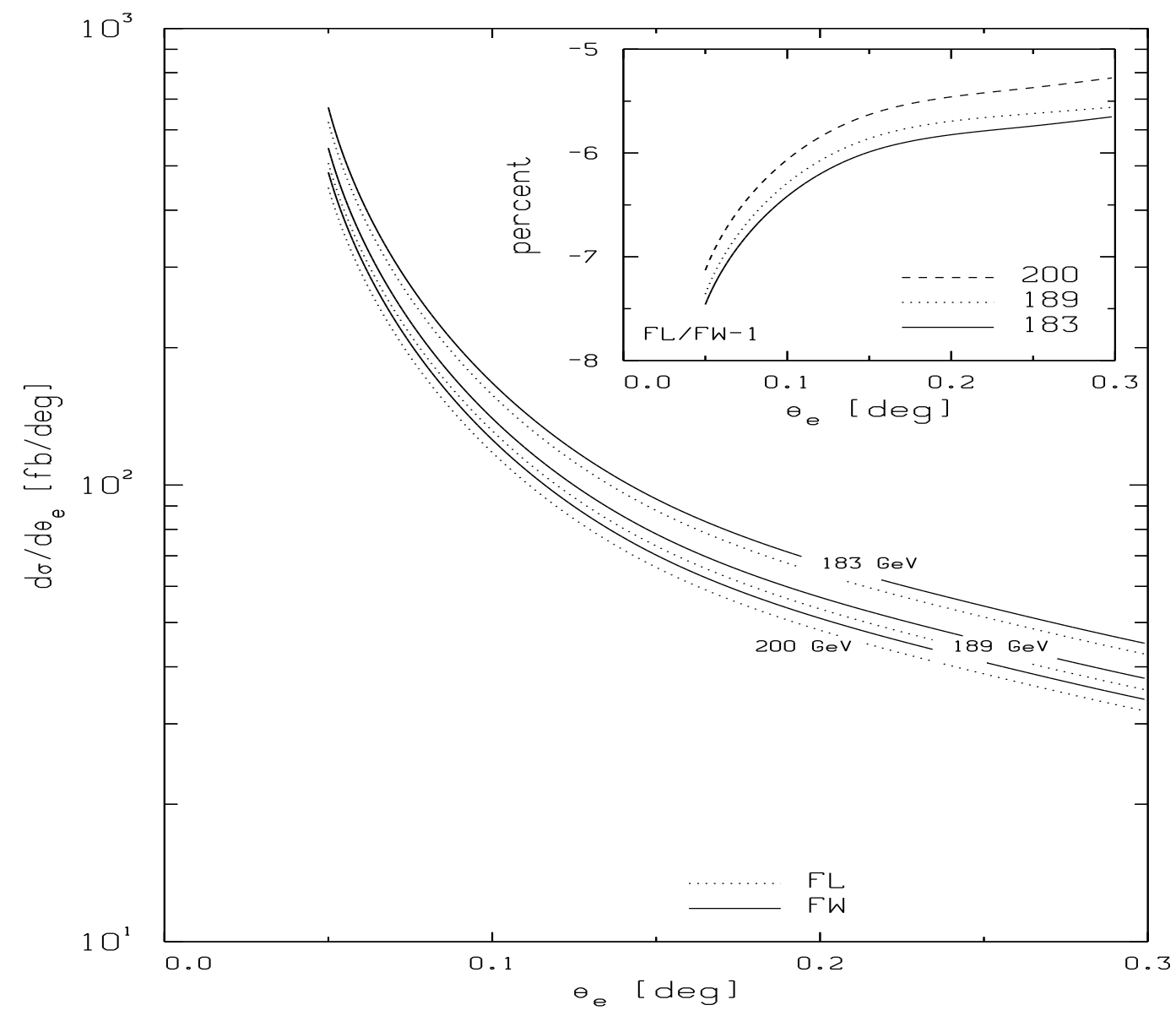

Figure 8: $d \sigma / d \cos \theta_{e}\left[\mathrm{fb} /\right.$ degrees] for $e^{+} e^{-} \rightarrow u \bar{d} e^{-} \bar{\nu}_{e}$ with $M(u \bar{d})>45 \mathrm{GeV}$ and $\sqrt{s}=$ $183,189,200 \mathrm{GeV}$.

between the FL distribution and the FW one for $\sqrt{s}=183 \mathrm{GeV}$. As before, only the most significant bins are shown $\left(0.0^{\circ} \div 0.4^{\circ}\right)$.

As for the hadronic case, the FL prediction is considerably lower than the FW one, although the percentage difference between the two is approximately $2.2 \% \div 2.4 \%$ smaller than in the previous case.

This fact is reflected in the total single- $W$ cross-section for $e^{+} e^{-} \rightarrow e^{-} \bar{\nu}_{e} \mu^{+} \nu_{\mu}$ that we report in Tab.(可).

When we want to improve upon the Fixed-Width scheme, the main accent has to be put on the correct evaluation of the scale in the running of $\alpha_{\mathrm{QED}}$. The latter is particularly important for the $t$-channel diagrams, dominated by a scale $q^{2} \approx 0$ and not $q^{2} \approx M_{W}^{2}$. However, a consistent implementation of radiative corrections does more than evolving $\alpha_{\mathrm{QED}}$ to the correct scale, other couplings are also running, propagators are modified and vertices are included.

We have performed a simple exercise, namely to take the Fixed-Width scheme augmented by a rescaling $\left[\alpha\left(q^{2}\right) / \alpha_{G_{F}}\right]^{2}$. Since the largest part of the effect is, in any case, confined in the region of very small electron scattering angle we only compute $d \sigma / d \theta_{e}$ for $0.0^{\circ}<\theta_{e}<0.4^{\circ}$. Results of our computation are reported in Tab.(6). From Tab.(6) we see that the effective FW-scheme describes considerably well the hadronic final state with a cut of $M(u \bar{d})>45 \mathrm{GeV}$, with a maximal difference of $0.85 \%$ in the first bin. However, the diminution induced by $\alpha_{\mathrm{QED}}\left(q^{2}\right)$ is too large for the leptonic final 


\begin{tabular}{|c||c|c|c|}
\hline$\theta_{e}[\mathrm{Deg}]$ & $\mathrm{FW}$ & $\mathrm{FL}$ & $\mathrm{FL} / \mathrm{FW}-1$ (percent) \\
\hline $0.0^{\circ} \div 0.1^{\circ}$ & 0.14154 & 0.13448 & -4.99 \\
\hline $0.1^{\circ} \div 0.2^{\circ}$ & 0.02113 & 0.02031 & -3.88 \\
\hline $0.2^{\circ} \div 0.3^{\circ}$ & 0.01238 & 0.01194 & -3.55 \\
\hline $0.3^{\circ} \div 0.4^{\circ}$ & 0.00880 & 0.00851 & -3.30 \\
\hline
\end{tabular}

Table 4: $d \sigma / d \theta_{e}$ in [pb/degrees] for the process $e^{+} e^{-} \rightarrow e^{-} \bar{\nu}_{e} \nu_{\mu} \mu^{+}$, for $\left|\cos \theta_{e}\right|>0.997, E_{\mu}>15 \mathrm{GeV}$, and $\left|\cos \theta_{\mu}\right|<0.95$. Furthermore, $\sqrt{s}=183 \mathrm{GeV}$.

\begin{tabular}{|c||c|c|c|}
\hline$\sqrt{s}$ & FW & FL & FL/FW-1 (percent) \\
\hline $183 \mathrm{GeV}$ & $26.77(14)$ & $25.53(4)$ & $-4.6(5)$ \\
$189 \mathrm{GeV}$ & $29.73(14)$ & $28.78(4)$ & $-3.2(5)$ \\
$200 \mathrm{GeV}$ & $36.45(23)$ & $34.97(6)$ & $-4.1(6)$ \\
\hline
\end{tabular}

Table 5: Total single- $W$ cross-section in fb for the process $e^{+} e^{-} \rightarrow e^{-} \bar{\nu}_{e} \mu^{+} \nu_{\mu}$, for $\left|\cos \theta_{e}\right|>0.997$, $E_{\mu}>15 \mathrm{GeV}$, and $\left|\cos \theta_{\mu}\right|<0.95$. The number in parenthesis shows the statistical error of the numerical integration on the last digit. 


\begin{tabular}{|c||c|c|c|c|c|c|}
\hline bin & FL & FWE & FL/FWE-1 (percent) & FL & FWE & FL/FWE-1 (percent) \\
\hline $0.0^{\circ} \div 0.1^{\circ}$ & 0.44784 & 0.45166 & +0.85 & 0.13448 & 0.13207 & -1.79 \\
\hline $0.1^{\circ} \div 0.2^{\circ}$ & 0.06605 & 0.06615 & +0.15 & 0.02031 & 0.01989 & -2.07 \\
\hline $0.2^{\circ} \div 0.3^{\circ}$ & 0.03860 & 0.03867 & +0.18 & 0.01194 & 0.01169 & -2.09 \\
\hline $0.3^{\circ} \div 0.4^{\circ}$ & 0.02736 & 0.02742 & +0.22 & 0.00851 & 0.00832 & -2.23 \\
\hline
\end{tabular}

Table 6: Comparison between FL-scheme and effective FW-scheme, i.e. FW augmented by the running of $\alpha_{\mathrm{QED}}$ in the $t$-channel. First set of results refers to $u \bar{d}$ final states. Second set to the $\mu^{+} \nu_{\mu}$ final state. Acceptance cuts are as in the main text and $\sqrt{s}=183 \mathrm{GeV}$.

state. The latter is a clear sign that other effects are relevant and a naive rescaling does not suffice in reproducing a realistic approximation in all situations, at least not within the $2 \%$ level of requested theoretical accuracy.

In order to understand this fact we have computed $d \sigma / d \theta_{e}$, in the first bin (largest effect), both for $u \bar{d}$ and for $\mu^{+} \nu_{\mu}$ final states with varying acceptance cuts. The results of this investigation are shown in Tab.(7) and Tab.(8), respectively. From the numbers in Tab.(7) we see the growth in the cross-section, for all schemes, due to the multi-peripheral peak. The percentage difference between FL-scheme and FW-scheme decreases, although slowly, for decreasing $M_{\min }(u \bar{d})$. There is more, the difference between the two schemes goes from $-12.91 \%$ for $35 \mathrm{GeV}<M(u \bar{d})<45 \mathrm{GeV}$ to $-6.96 \%$ for $25 \mathrm{GeV}<M(u \bar{d})<35 \mathrm{GeV}$, to $-3.58 \%$ for $15 \mathrm{GeV}<M(u \bar{d})<25 \mathrm{GeV}$ and reaches $-1.20 \%$ for $5 \mathrm{GeV}<M(u \bar{d})<10 \mathrm{GeV}$. With the above cuts we are removing the contribution from the single-resonant $W$-exchange and the multi-peripheral diagrams start dominating. The same trend is clearly visible in Tab.(8), despite the somewhat larger errors. By increasing $\cos \theta_{\mu \text {,min }}$ we allow the muon to become more and more collinear to the quasi-real photon, therefore the multi-peripheral contribution counts more and more.

For the multi-peripheral diagrams alone, the inclusion of fermionic corrections changes $\alpha \rightarrow \alpha\left(\left(p-{ }_{-}\right.\right.$ $\left.\left.q_{-}\right)^{2}\right)$ in the $t$-channel photon exchange, $g^{2} \rightarrow g^{2}\left(\left(p_{+}-q_{+}^{2}\right)^{2}\right)$ in the $t$-channel $W$ exchange and modifies the $t$-channel $W$-propagator. The latter is a large effect. To see it, let us define $t$-channel line-shape functions,

$$
\begin{aligned}
L_{\mathrm{FL}}(t) & =\frac{s^{2}}{\left(t-\operatorname{Re} p_{W}\right)^{2}+\left(\operatorname{Im} p_{W}\right)^{2}} \frac{1}{|R(t)|^{2}}, \\
L_{\mathrm{FW}}(t) & =\frac{s^{2}}{\left(t-M_{W}^{2}\right)^{2}+M_{W}^{2} \Gamma_{W}^{2}} .
\end{aligned}
$$

and compare them, as shown in Fig. 9. The FL $t$-channel line-shape can be considerably larger than 


\begin{tabular}{|c||c|c|c|}
\hline$M_{\min }(u \bar{d})[\mathrm{GeV}]$ & $\mathrm{FL}$ & $\mathrm{FW}$ & $\mathrm{FL} / \mathrm{FW}-1$ (percent) \\
\hline 45 & $0.04478(3)$ & $0.04841(3)$ & $-7.5(1)$ \\
35 & $0.04711(6)$ & $0.5104(7)$ & $-7.7(1)$ \\
25 & $0.504(1)$ & $0.0546(1)$ & $-7.7(2)$ \\
15 & $0.0552(1)$ & $0.0595(1)$ & $-7.2(2)$ \\
10 & $0.0582(1)$ & $0.0626(1)$ & $-7.0(2)$ \\
5 & $0.0615(1)$ & $0.0659(1)$ & $-6.7(2)$ \\
1 & $0.0637(1)$ & $0.0682(1)$ & $-6.6(2)$ \\
\hline
\end{tabular}

Table 7: Comparison between FL-scheme and FW-scheme for the cross-section in pb and $0.0^{\circ}<$ $\theta_{e}<0.1^{\circ}$. Furthermore, $M(u \bar{d}) \geq M_{\min }$ and $\sqrt{s}=183 \mathrm{GeV}$. The number in parenthesis shows the statistical error of the numerical integration on the last digit.

\begin{tabular}{|c||c|c|c|}
\hline $\cos \theta_{\mu, \min }$ & $\mathrm{FL}$ & $\mathrm{FW}$ & $\mathrm{FL} / \mathrm{FW}-1$ (percent) \\
\hline 0.95 & $0.01345(1)$ & $0.01415(1)$ & $-4.9(1)$ \\
0.995 & $0.01485(9)$ & $0.01538(4)$ & $-3.4(6)$ \\
0.9995 & $0.01543(13)$ & $0.01571(9)$ & $-1.8(9)$ \\
\hline
\end{tabular}

Table 8: Comparison between FL-scheme and FW-scheme for the cross-section in pb, $0.0^{\circ}<\theta_{e}<$ $0.1^{\circ},\left|\cos \theta_{\mu}\right|<\cos \theta_{\mu, \min }$. Furthermore $\sqrt{s}=183 \mathrm{GeV}$. The number in parenthesis shows the statistical error of the numerical integration on the last digit. 


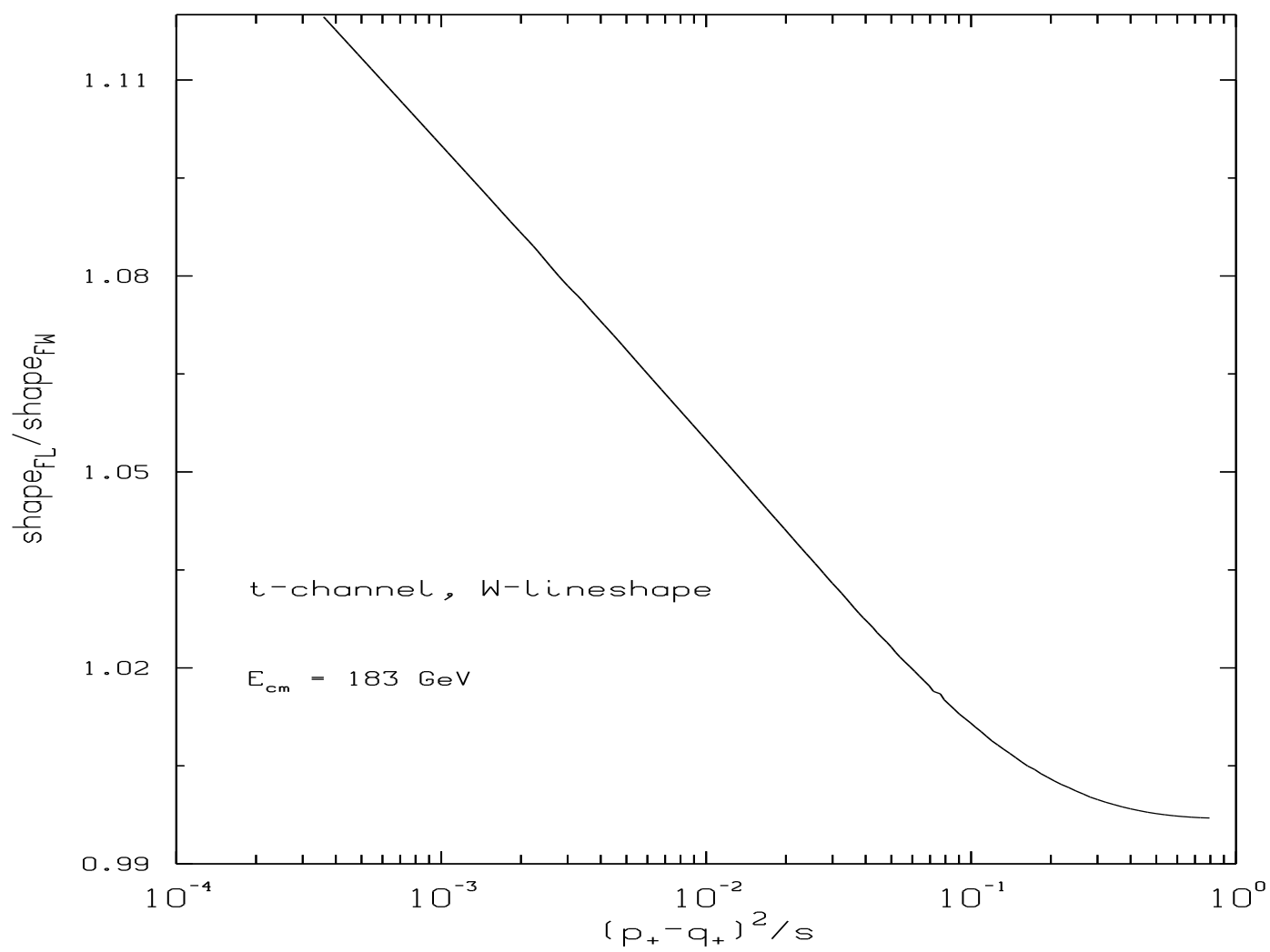

Figure 9: Comparison between the Fixed-Width and the Fermion-Loop line-shape functions in the t-channel, Eq.(31).

the FW one, due to the $\rho$-factor, $R(t)$, of Eq.(15). In a circumstance where vertices and $s$-channel components count very little we can simulate the three effects induced by the FL-scheme in a simple way. To this end, we compare the following objects:

$$
\begin{aligned}
\mathrm{FL}_{\text {eff }}(t, T) & =\alpha^{2}(T)\left|g^{2}(t)\right|^{2} \frac{s^{2}}{\left(t-\operatorname{Re} p_{W}\right)^{2}+\left(\operatorname{Im} p_{W}\right)^{2}} \frac{1}{|R(t)|^{2}} \\
\mathrm{FW}_{\mathrm{eff}}(t) & =g^{8} s_{\theta}^{4} \frac{s^{2}}{\left(t-M_{W}^{2}\right)^{2}+M_{W}^{2} \Gamma_{W}^{2}}
\end{aligned}
$$

where $T=-\left(p_{-}-q_{-}\right)^{2}$ and $t=-\left(p_{+}-q_{+}\right)^{2}$. The comparison is shown, for fixed $T$ and as a function of $t$, in Fig. 10. The difference, especially for non-vanishing values of $t$ (remember that $M_{W}^{2} / s=0.19$ at $\sqrt{s}=183 \mathrm{GeV}$ ), is considerably smaller than the one caused by the running of $\alpha_{\mathrm{QED}}$. This confirms our previous findings and gives clear evidence that a naive rescaling of the Fixed-Width results cannot cover all possible cases, with different processes and with kinematical cuts of different kinds, at least not below the threshold of $2 \%$ for the theoretical accuracy.

Modifications induced by the fermionic loops are sensitive to the relative weight of single-resonant terms and of multi-peripheral peaks. Furthermore, the effect of radiative corrections inside the $W$ propagators ( $\rho$-factors) is far from being negligible and tends to compensate the change due to the 


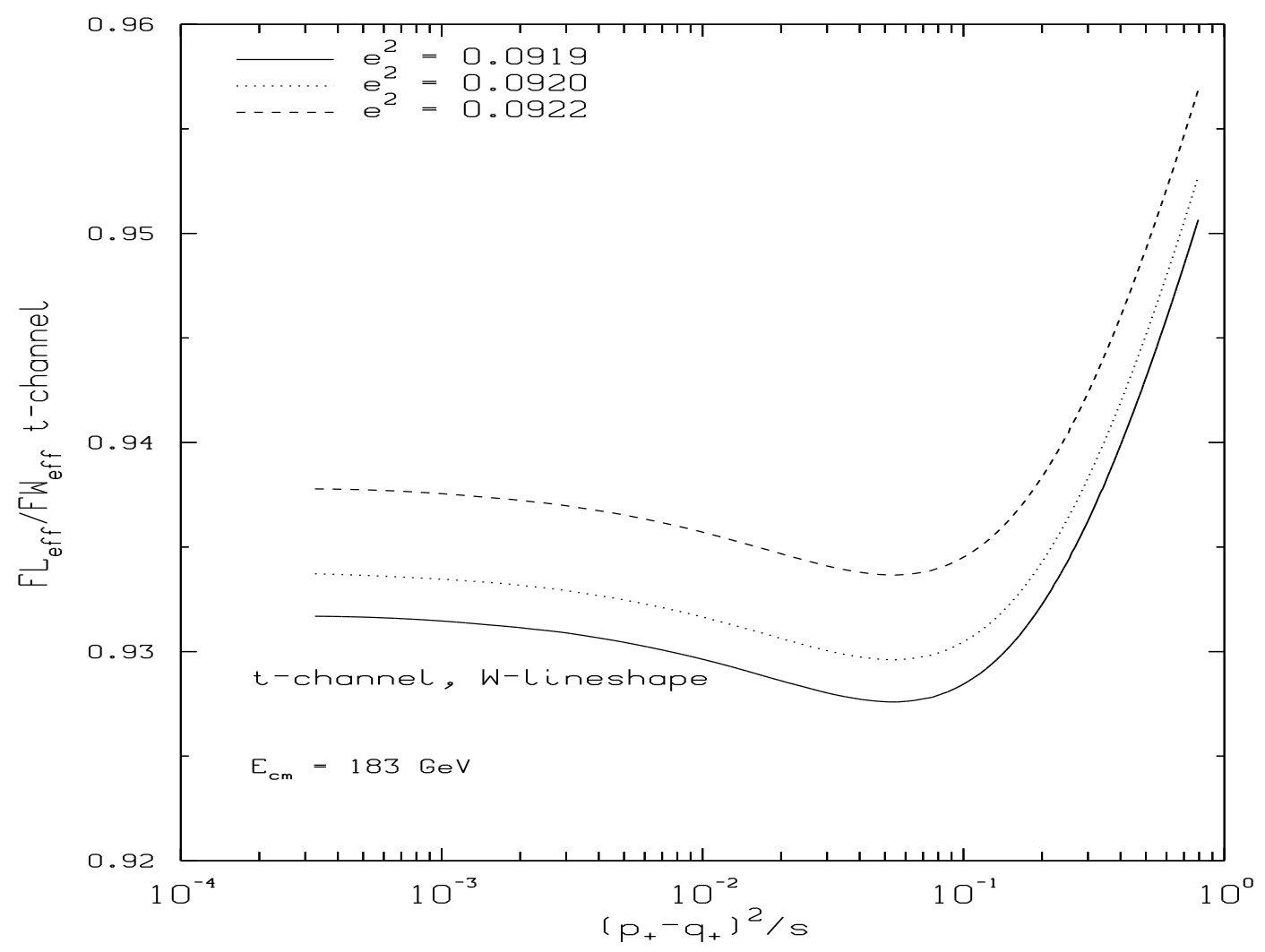

Figure 10: Comparison between the fixed-width and the fermion-loop line-shape functions in the $t$-channel, Eq.(32). Three values of $e^{2}(T)$ are reported.

running of $\alpha_{\mathrm{QED}}$.

\section{Conclusions.}

In this paper we have analyzed the numerical impact of introducing the Fermion-Loop scheme for evaluating cross-sections and distributions in single- $W$ production.

A description of single- $W$ processes by means of the Fermion-Loop scheme is mandatory from, at least, two points of view. FL is the only field-theoretically consistent scheme that preserves gauge invariance in processes including unstable vector-bosons coupled to e.m. currents. Furthermore, single$W$ production is a process that depends on several scales, the single-resonant $s$-channel exchange of $W$-bosons, the exchange of $W$-bosons in $t$-channel, the small scattering angle peak of outgoing electrons.

A correct treatment of the multi-scale problem can only be achieved when we include radiative corrections in the calculation, not only one-loop terms but also the re-summation of leading higherorder terms. Recent months have shown that this project can be brought to a very satisfactory level by identifying the correct approximation, process-by-process.

In particular, the $W-W$ configuration, dominated by double-resonant terms, can be treated within 
the so-called double-pole-approximation or DPA [18]. As a consequence, the theoretical uncertainty associated with the determination of the $W W$ cross-section is sizably decreased. In principle, the same procedure applies to the determination of the $Z Z$ cross-section, where one develops a NC02DPA approximation instead of the CC03-DPA one.

\begin{tabular}{|c||c|c|c|}
\hline Process & $\sqrt{s}=183 \mathrm{GeV}$ & $\sqrt{s}=189 \mathrm{GeV}$ & $\sqrt{s}=200 \mathrm{GeV}$ \\
\hline$e^{+} e^{-} \rightarrow e^{-} \bar{\nu}_{e} u \bar{d}$ & $83.237(36)$ & $93.797(75)$ & $113.71(5)$ \\
\hline$e^{+} e^{-} \rightarrow e^{-} \bar{\nu}_{e} \nu_{\mu} \mu^{+}$ & $25.476(40)$ & $28.741(44)$ & $34.900(50)$ \\
\hline$e^{+} e^{-} \rightarrow e^{-} \bar{\nu}_{e} \nu_{\tau} \tau^{+}$ & $25.442(38)$ & $28.702(41)$ & $34.856(48)$ \\
\hline
\end{tabular}

Table 9: Single- $W$ cross-sections for $\sqrt{s}=183(189,200)$ GeV and $\left|\cos \theta_{e}\right|>0.997$ in the FermionLoop scheme. Additional cuts are: $M(u \bar{d})>45 \mathrm{GeV} ; E_{\mu(\tau)}>15 \mathrm{GeV} ;\left|\cos \theta_{\mu(\tau)}\right|<0.95$. The number in parenthesis shows the statistical error of the numerical integration on the last two digits.

In this paper we have considered the single- $W$ production, i.e. $e^{+} e^{-} \rightarrow W e \nu$. Fermionic loops have been included, following the generalization of the Fermion-Loop scheme worked out in [9]. Within this context theoretical predictions for single- $W$ production become smaller, as compared to other pragmatic, gauge-preserving, schemes. We have found that all the modifications introduced via the Fermion-Loop scheme are relevant: running of the couplings, $\rho$-factors and vertices, not only the change $\alpha_{\mathrm{QED}}$ (fixed) $\rightarrow \alpha_{\mathrm{QED}}$ (running). Therefore, a naive rescaling cannot reproduce the FermionLoop answers for all situations, all kinematical cuts.

The high-energy Input Parameter Set used in all calculations that are presently available - we quote, among the various schemes, the Fixed-Width scheme, the Overall scheme and the Imaginary Fermion-Loop one - is based on $G_{F}, M_{W}$ and $M_{Z}$ with $\alpha_{\mathrm{QED}}$ (fixed) $=1 / 131.95798$. It allows for the inclusion of part of higher order effects in the Born cross-sections but, it fails to give a correct and accurate description of the $q^{2} \sim 0$ dominated processes.

A naive, overall, rescaling would lower the single- $W$ cross-section of about $7 \%$. We have found, with the complete Fermion-Loop, that this decrease is process and cut dependent. Moreover, the effect is larger in the first bin for $\theta_{e}-0.0^{\circ} \div 0.01^{\circ}$ - in the distribution $d \sigma / d \theta_{e}$ and tends to become less pronounced for larger scattering angles of the electron. However, the first bin represents almost $50 \%$ of the total single- $W$ cross-section, so that, in general, the compensations that occur among several effects never bring the $\mathrm{Fl} / \mathrm{FW}$ ratio to one. We obtain a maximal decrease of about $7 \%$ in the result but, on average, the effect is smaller. We have also found that the effect is rather sensitive to the relative weight of multi-peripheral contributions.

A final comment is needed to quantify the theoretical accuracy of single- $W$ production. Bosonic corrections are still missing and, very often, our experience has shown, especially at LEP1, that bosonic corrections may become sizeable [19]. A large part of the bosonic corrections, as e.g. the leading- 
logarithmic corrections, factorize and can be treated by a convolution. Nevertheless the remaining bosonic corrections can still be non-negligible, i.e., of the order of one percent at LEP2 [20]. For the Born cross-sections 1\% should, therefore, be understood as the present limit for the theoretical uncertainty. This will have to be improved, soon or later, since bosonic corrections are even larger at higher energies [21] and the single- $W$ cross-section will cross over the $W W$ one at $500 \mathrm{GeV}$. Single- $W$ will be one of the major processes at LC to measure not only triple gauge couplings but also the $W$-mass without color reconnection.

To summarize our findings we present a collection of single- $W$ cross-sections obtained in the Fermion-Loop scheme with WTO in a high precision run. They are shown in Tab.(9) where we also report the $\nu_{\tau} \tau$ final state. Numerical precision ranges from $0.05 \% \div 0.08 \%$ for $u \bar{d}$ to $0.14 \% \div 0.16 \%$ for $\nu_{\mu} \mu\left(\nu_{\tau} \tau\right)$.

\section{Acknowledgements.}

Over the last year I had a lot of fruitful discussions with many experimental colleagues and I profited a lot from their experience. In particular I want to mention the work of Martin Grünewald, Hywel Phillips, Alexander Schmidt-Kaerst, Reisaburo Tanaka and Marco Verzocchi. I also acknowledge many discussions with the participants in the LEP2/MC workshop, in particular Alessandro Ballestrero, Misha Dubinin, Roberto Pittau, Yoshimasa Kurihara and Maciej Skrzypek. 


\section{References}

[1] G. Passarino, The Single W Production Case, hep-ph/9810416.

[2] E. Boos and M. Dubinin, hep-ph/9909214;

C. G. Papadopoulos, in Muenchen/Annecy/Hamburg 1992-93, $e^{+} e^{-}$collisions at 500-GeV, pt. C 199-205;

M. A. Doncheski, S. Godfrey and K. A. Peterson, hep-ph/9710299 ;

T. Tsukamoto, hep-ph/9611212, Acta Phys. Polon. B28(1997)695;

T. Tsukamoto and Y. Kurihara, hep-ph/9607353, Phys. Lett. B389(1996)162;

K. Huitu, J. Maalampi and M. Raidal, hep-ph/9602387;

C. G. Papadopoulos, hep-ph/9402208, Phys.Lett. B333(1994)202.

[3] ALEPH Collaboration (R. Tanaka for the collaboration). CERN-OPEN-99-280, Jul 1999;

ALEPH Collaboration (R. Barate et al.). CERN-EP-99-086, Jun 1999, Phys.Lett. B462(1999)389;

R. Tanaka, X-LPNHE-98-08, In Vancouver 1998, High energy physics, vol. 1 515-519. hepex/9811039;

L3 Collaboration (M. Acciarri et al.). CERN-EP-98-099, Jun 1998, Phys. Lett. B436(1998)417;

L3 Collaboration (M. Acciarri et al.). CERN-PPE-97-028, Mar 1997, Phys. Lett. B403(1997)168.

[4] D. Bardin et al. Report on Event Generators for $W W$ Physics in Vol.1, 'Report of the Workshop in Physics at LEP2', G. Altarelli et. al., CERN-96-01.

[5] E. Argires et al., Phys. Lett. B358(1995)339.

[6] Y. Kurihara, D. Perret-Gallix and Y. Shimizu, Phys. Lett. B349(1995) 367.

[7] W. Beenakker, G. J. van Oldenborgh, A. Denner, S. Dittmaier, J. Hoogland, R. Kleiss, C. G. Papadopoulos and G. Passarino, hep-ph/9612260, Nucl. Phys. B500(1997)255.

[8] U. Baur and D. Zeppenfeld, Phys. Rev. Lett. 75(1995)1002.

[9] G. Passarino, Unstable Particles and Non-Conserved Currents: A Generalization of the FermionLoop Scheme, hep-ph/9911482.

[10] J. Hoogland and G. J. van Oldenborgh, Phys. Lett. B402(1197)379.

[11] G. Passarino, Comp. Phys. Comm. 97(1996)261.

[12] F. Berends, R. Kleiss and R. Pittau, Comp. Phys. Comm. 85(1995)437;

F. Caravaglios and M. Moretti, Phys. Lett. B308(1995)332;

G. Passarino, Comp. Phys. Comm. 97(1996)261;

J. Fujimoto et al., Comp. Phys. Comm. 100(1997)128;

D. G. Charlton et al., Comp. Phys. Comm. 99(1997)335;

E. Accomando and A. Ballestrero, Comp. Phys. Comm. 99(1997)270;

A. Pukhov et al, hep-ph/9908288;

S. Jadach et al., Comp. Phys. Comm. 119(1999)272.

[13] A. Kanaki and C .G. Papadopoulos, private communication and work in preparation.

[14] E. Accomando, A. Ballestrero and E. Maina, hep-ph/9911489. 
[15] D. Bardin and G. Passarino, The Standard Model in the Making, Clarendon Press - Oxford, 1999.

[16] G. Passarino and M. Veltman, Nucl. Phys. B160(1979)151.

[17] C. Caso et al., Euro. Phys. Jour. C3(1998)1.

[18] A. Denner, S. Dittmaier, M. Roth and D. Wackeroth, BI-TP-99-47, Dec 1999, hep-ph/9912447; A. Denner, S. Dittmaier, M. Roth and D. Wackeroth, BI-TP-99-46, Dec 1999, hep-ph/9912290;

A. Denner, S. Dittmaier, M. Roth and D. Wackeroth, BI-TP-99-45, Dec 1999, 9pp. hepph/9912261;

S. Jadach, W. Placzek, M. Skrzypek, B. F. L. Ward and Z. Was, hep-ph/9907436

S. Jadach, W. Placzek, M. Skrzypek, B. F. L. Ward and Z. Was Comput.Phys.Commun. 119:272311,1999, hep-ph/9906277;

S. Jadach, W. Placzek, M. Skrzypek, B. F. L. Ward and Z. Was Phys.Lett.B417:326-336,1998, hep-ph/9705429.

[19] D. Bardin, W. Hollik and G. Passarino (eds.), Reports of the working group on precision calculations for the $Z$ resonance (CERN 95-03, Genève, 1995).

[20] W. Beenakker et al., in Physics at LEP2, eds. G. Altarelli, T. Sjöstrand and F. Zwirner, (CERN 96-01, Genève, 1996) Vol. 1, p. 79, hep-ph/9602351.

[21] P.M. Zerwas (ed.), $e^{+} e^{-}$collisions at $500 \mathrm{GeV}$ : The physics potential (DESY 93-123C, Hamburg, 1993);

W. Beenakker and A. Denner, Int. J. Mod. Phys. A9 (1994) 4837. 